\title{
An Overview of Radio Resource Management in Relay-Enhanced OFDMA-Based Networks
}

\author{
Mohamed Salem, Abdulkareem Adinoyi, Mahmudur Rahman, Halim Yanikomeroglu, David Falconer, \\ Young-Doo Kim, Eungsun Kim, and Yoon-Chae Cheong
}

\begin{abstract}
Researchers in both academia and industry have accepted OFDMA as the most appropriate air-interface for the emerging broadband wireless access networks and standards. A number of IEEE working groups and various research forums are focusing on developing relay and mesh-enabled networks with cooperative communication features. Among these research efforts are IEEE $802.11 \mathrm{~s}$, IEEE $802.16 \mathrm{j} / \mathrm{m}$, and 3GPP's advanced long term evolution (LTE-advanced). The combination of OFDMA with relaying techniques provides rich opportunities for cost-effective and high-performance networks. To exploit such opportunities requires intelligent radio resource management (RRM) algorithms.

Although a number of publications have highlighted the important and challenging issues involved in designing RRM algorithms for OFDMA networks, only recently a number of papers have investigated relay-enhanced OFDMA-based multicellular networks. By and large, the literature indicates that these issues constitute a hot research topic that will continue to attract interest. This paper provides a survey of the current literature on OFDMA networks enhanced with decode-and-forward relaying and provides their link to earlier literature in non-OFDMA networks. In addition, a rich list of references is provided to direct the readers toward some of the emerging techniques.
\end{abstract}

Index Terms-RRM, OFDMA, relaying, 4G, scheduling, throughput, fairness.

\section{INTRODUCTION}

$\mathbf{N}$ EXT-GENERATION wireless communication networks are expected to provide ubiquitous high-data-rate coverage in the most cost-effective manner. To achieve this objective, high-spectral-efficiency schemes are required in conjunction with aggressive resource reuse strategies to ensure prudent use of scarce radio resources. Interest in orthogonal frequency-division multiplexing (OFDM) is growing steadily, as it appears to be a promising air-interface for the next generation of wireless systems due, primarily, to its inherent resistance to frequency-selective multi-path fading and the flexibility it offers in radio resource allocations. Exploiting the dynamism in wireless channels, OFDM subcarriers can be adaptively modulated and/or assigned to the "best" wireless

Manuscript received 6 October 2008; revised 13 February 2009 and 12 May 2009. This work was supported by Samsung Electronics Co. Ltd., SAIT, Korea.

M. Salem, M. Rahman, H. Yanikomeroglu, and D. Falconer are with the Department of Systems and Computer Engineering, Carleton University, Ottawa, Canada (e-mail: \{mrashad, halim, ddf $\}$ @sce.carleton.ca).

A. Adinoyi is currently with Swedtel Arabia (email: adinoyi@sce.carleton.ca).

Y.-D. Kim, E. Kim, and Y.-C. Cheong are with Samsung Electronics, SAIT, Korea (e-mail: \{yd76.kim, eungsun.kim, yc.cheong\}@samsung.com).

Digital Object Identifier 10.1109/SURV.2010.032210.00071 subscriber (WS) to achieve frequency and multi-user diversity gains. OFDMA results from using OFDM in conjunction with frequency-division multiple access protocol, in which a WS may be assigned one or more subcarriers in order to meet its communication requirements.

Wireless coverage is only ubiquitous if reliable service is provided throughout the serving area of a base station (BS). It is advantageous for network service providers to distribute system capacity across the network area, reaching WSs in the most cost-effective way. With the traditional cellular architecture, increasing the capacity along with the coverage requires the deployment of a large number of BSs. This approach is cost prohibitive to network service providers [1], [2]. As an alternative, relaying techniques are expected to alleviate this coverage problem since the relay station (RS) with less functionality than the BS can forward high data rates to remote areas of the cell while lowering infrastructure cost. The synergy between OFDMA and relaying techniques offers a promising technology for providing high data rate to WSs everywhere, anytime.

To this end, an OFDMA-based relay-enhanced network comprising various forms of infrastructure-based or dedicated relays is envisaged in the next-generation networks. These relays could be owned, leased or given at no cost to WSs by the service provider. How to perform radio resource management (RRM) in such a complex environment is not immediately clear. However, there is a consensus on the need to devise intelligent RRM algorithms with excellent bandwidth efficiency that can harness the full potentials of relay-enhanced OFDMA-based networks. The literature reveals that RRM algorithms have been extensively investigated for OFDMAbased networks without relays, e.g., [3] - [7] and surveyed in [8]; it is only recently that attention has started to shift to relay-based OFDMA multi-cell networks.

This paper provides an overview on the recent developments in RRM algorithms in OFDMA-based wireless networks enhanced with fixed relays employing decode-and-forward relaying technique. Figure 1 shows a partial network in a multicellular relay network. The RRM problem example discussed in Section III and the numerical results in Section VIII are based on this figure.

The figure also shows that the BS could continuously measure the quality of link, e.g., signal-to-interference-plus noise ratio (SINR) per subcarrier. For slowly varying channels, BSs and RSs can be assumed to have accurate estimate of the channel states. The BSs can optimize the resource allocation 


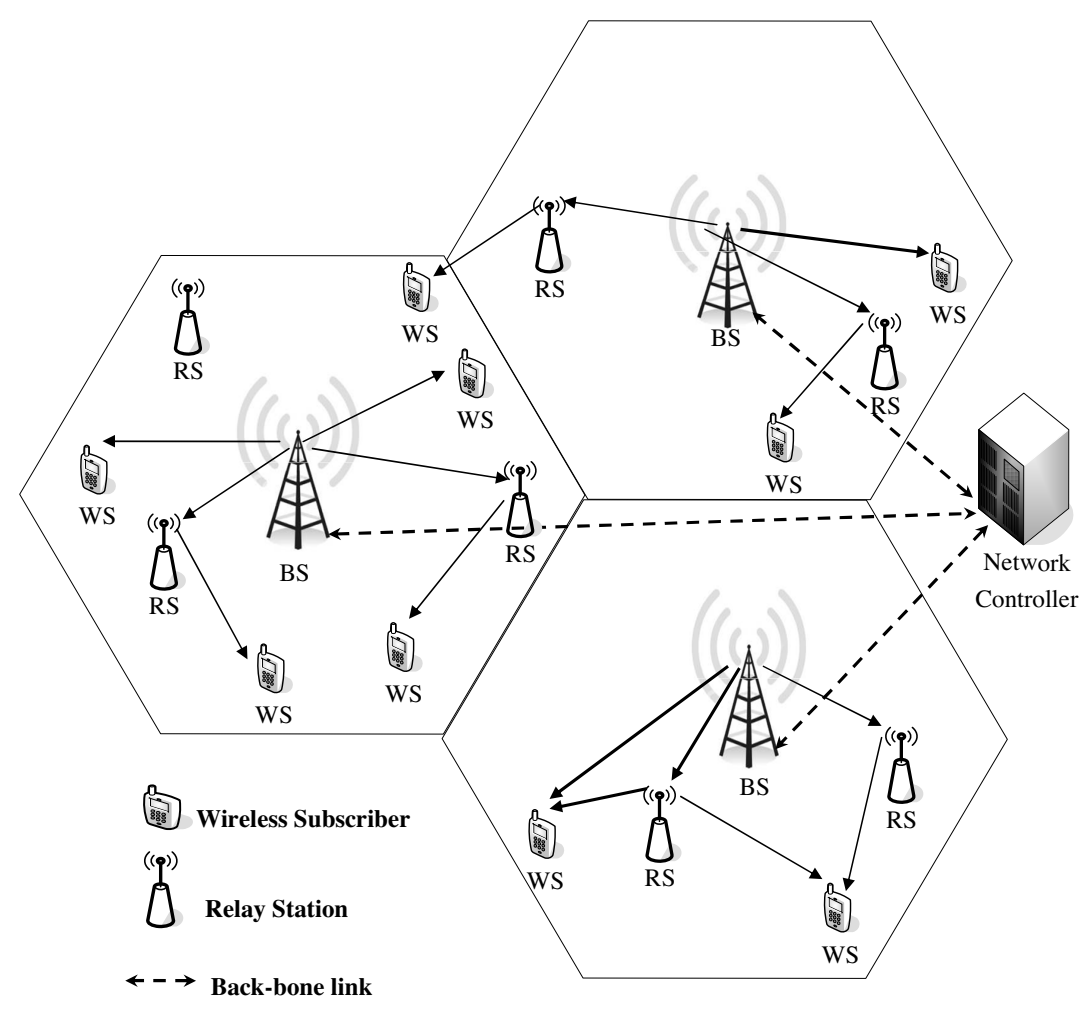

Fig. 1. A partial network in multicellular relay networks.

or they can involve a central or network controller as shown in Fig. 1. The radio resources are assigned to links based on a number of considerations with the overall aim of improving the throughput. Fairness among WSs can also be an important system performance to improve. The BS directly or through RSs serves the WSs in each cell. The data of each WS in a cell can potentially be routed through any of the relays according to the routing criterion. In networks implementing global frequency reuse, all cells share the same spectrum. The total bandwidth is divided into $N$ subchannels each composed of a set of adjacent OFDM data subcarriers. It is also observed that cooperation (where a WS is served through more than one link) can be invoked to deliver a reliable service to WSs.

The rest of this paper is organized as follows: The ongoing research activities in standardization bodies and forums in the context of relaying are discussed in Section II. An RRM optimization statement and techniques are presented in Section III. Earlier RRM literature in non-OFDMA relay networks is discussed in Section IV and approaches for performing resource allocation in OFDMA-based fixed relay networks are given in Section V. Optimization techniques and complexity issues are mentioned in Section VI. Fairness metrics are briefly discussed in Section VII. Channel models and numerical examples are presented in Section VIII and finally, conclusion is given in Section IX.

Readers are also referred to [9] in which we focus on some of the prominent challenges involved in migrating from the conventional cellular architecture to the relay-based type and further discuss how intelligent RRM schemes can exploit the opportunities in relay-enhanced OFDMA-based cellular networks.

\section{ACTIVITIES ON RELAY-BASED ARCHITECTURE AMONG STANDARDIZATION BODIES, FORUMS, AND CONSORTIUMS}

To the authors' knowledge, the first standard involving relays in the form of analog repeaters was introduced in personal wireless terminal (PWT) interoperability standard in the 90's. PWT emerged from ETSI digital enhanced cordless telecommunications (DECT) standard as a North American TIA variant. Operated in the $1900 \mathrm{MHz}$ band using timedivision duplex (TDD) and time-division multiple access (TDMA), these relays were to extend range up to a few kilometers for voice and low-rate data services [10]. In recent years, as one of the key technologies for $4 \mathrm{G}$ networks, relaying has again attracted tremendous attention in order to obtain coverage/capacity enhancement as well as to extract benefits such as cooperative diversity gains.

The idea of relaying has previously been examined in the 3GPP in the form of ad-hoc peer-to-peer multi-hop protocol known as opportunity-driven multiple access (ODMA). In the ODMA protocol, the cell-edge WSs communicate with the BS through the help of other WSs that are closer to the BS. However, the ODMA protocol did not make it to the standard due to complexity and signalling overhead concerns [11]. Nevertheless, a number of lessons were learnt. Currently, numerous standardization bodies, forums, and consortiums have been working on relay-based architectures in scenarios ranging from wireless local area networks (WLAN) to widearea cellular networks.

Early WLAN standards, such as IEEE $802.11 \mathrm{a} / \mathrm{b} / \mathrm{g}$, do not support relay-based deployment due to their contention-based multiple access technique. Researchers proposed a number of possible relay-friendly modified medium access control 
protocols, for instance, relay-enabled point coordination function ( $\mathrm{rPCF})[12]$ and relay-enabled distributed coordination function $(\mathrm{rDCF})$ [13], among others, to enable the use of relays with these standards. However, Task Group "S" of the IEEE 802.11 Work Group, formed in 2003, released an unapproved draft version of IEEE 802.11s wireless mesh network in March 2007, which is an amendment to support mesh and relay based WLAN. The key element in this WLAN version is called mesh point (MP). Being very similar to the traditional access point (AP), MP can connect mesh APs, other MPs, and STAtions (STA) wirelessly. An excellent overview on 802.11s is available in [14]. European ETSI HIPERLAN/2, on the other hand, has shown an ability to support relay-based deployment due to its dynamic TDMA access on OFDM airinterface.

IEEE 802.16m and 3GPP advanced long term evolution (LTE-A), which contend beyond $3 \mathrm{G}$ standards are the two most notable standards to advocate relaying. Although IEEE $802.16 \mathrm{~m}$ inherits multi-hop relaying from predecessor IEEE 802.16j standard, the evolving LTE-A is expected to incorporate multi-hop relaying to further narrow down the gaps between these two standards. The worldwide interoperability for microwave access (WiMAX) forum was created in 2001 to promote conformance and interoperability of products based on harmonized IEEE 802.16/ETSI HiperMAN standard. Like IEEE WLAN, the focus of IEEE 802.16 early standards was not on relaying until IEEE 802.16e; in fact, this is the first standard that supports mobility along with basic mesh networking capability. IEEE $802.16 \mathrm{j}$ mobile multi-hop relay is an amendment to IEEE 802.16e standard focusing on coverage/capacity expansion. Recent unapproved draft version of $802.16 \mathrm{j}$ has been released in February 2009 [16]. In IEEE $802.16 \mathrm{~m}$, OFDMA in TDD has been used for both the downlink and uplink while OFDMA and single carrier frequency-division multiple access are the access schemes for the downlink and uplink of LTE, respectively. Adaptive OFDMA is proposed for the downlinks in 3GPP-LTE or Evolved UTRA. To this end, an excellent overview on design issues relating to adaptive transmission for OFDMA-based systems beyond 3G can be found in [15].

In addition to these standardization efforts, European $6^{\text {th }}$ framework projects such as FIREWORKS ${ }^{1}$ and Wireless World Initiative New Radio (WINNER ${ }^{2}$ ) have been involved in the 4G (and beyond) wireless research. They consider relaying and OFDMA as essential elements required to meet the demand of future wireless services. Accordingly, RRM design occupies a pivotal position in these projects.

\section{The RRM Problem StATEMENT}

RRM algorithms are usually designed to exploit the variations in wireless channels by adaptively distributing scarce communication resources to either maximize or minimize some network performance metrics. In relay-enhanced OFDMA-based wireless networks, a typical resource allocation problem statement might be stated as follows:

\footnotetext{
${ }^{1}$ http://www.ist-fireworks.eu

${ }^{2}$ www.winner-ist.org
}

"How many information bits, how much transmit power (for WSs, relays, or BS), and how many subcarriers (or subchannels) should be assigned to WSs to either maximize or minimize a desired performance metric, e.g., system throughput (capacity) or total transmit power in the network, respectively?"

In a situation where a network device has "free" access to energy (e.g., fixed relay applications, where off-the-wall power is always available), the main objective of the RRM algorithm in downlink transmissions could simply be to maximize the system throughput. To this end, scheduling, routing, bitloading and adaptive modulation constitute some of the tools that are commonly employed in RRM solutions.

An Optimization Example [17]: Let us consider formulating the downlink sum-rate-maximizing subcarrier allocation problem for a half-duplex relay network, assuming that the average power $(P)$ for each subcarrier is the same and fixed. Consider a single cell from the network shown in Fig. 1 where the WS and RS are treated as contending for radio resources, an optimization problem can be expressed in the following way. Let $M, K$, and $N$ represent the number of relays, WSs, and subchannels, respectively. Let $\rho_{m, n}$ represent subcarrier $n$ assignment variable, indicating whether subcarrier $n$ is allocated to the $\mathrm{BS}-\mathrm{RS}_{m}$ link $(m=1, \cdots, M)$ or BS $\mathrm{WS}_{m}$ link $(m=M+1, \cdots, M+K)$. Let $\rho_{m, k, n}^{*}$ also indicate that subcarrier $n$ is allocated to the $\mathrm{BS}(m=0)$ or $\mathrm{RS}_{m}$ ( $m=1, \cdots, M)-\mathrm{WS}_{k}$ link, during the following time slot of the downlink frame. Let $\gamma_{m, n}$ and $\gamma_{m, k, n}^{*}$ denote the signalto-interference-plus-noise ratio (SINR) in the corresponding transmissions. Assuming that the channel does not change, we can express $\gamma_{M+j, n}=\gamma_{0, j, n}^{*}(j=1, \ldots, K)$, which will lead to the subcarrier allocation problem formulated in [17] as:

$$
\max _{\boldsymbol{\rho}, \boldsymbol{\rho}^{*}} \frac{1}{2} \sum_{m=1}^{M} \min \left\{A_{m}, B_{m}\right\}+\frac{1}{2}\left(\sum_{m=M+1}^{M+K} A_{m}+B_{0}\right),
$$

subject to:

$$
\begin{gathered}
\sum_{m=1}^{M+K} \rho_{m, n}=1, \forall n, \\
\sum_{m=0}^{M} \sum_{k=1}^{M+K} \rho_{m, k, n}^{*}=1, \forall n, \\
\rho_{m, n} \in\{0,1\}, \forall m, n, \\
\rho_{m, k, n}^{*} \in\{0,1\}, \forall m, k, n,
\end{gathered}
$$

where $A_{m}=\sum_{n=1}^{N} \rho_{m, n} \log _{2}\left(1+\gamma_{m, n} P\right)$ and $B_{m}=\sum_{n=1}^{N} \sum_{k=1}^{K} \rho_{m, k, n}^{*} \log _{2}\left(1+\gamma_{k, m, n}^{*} P\right)$.

The first term in the cost function (1) represents the achievable rate of data flow from BS to WSs via RSs and the second term stands for the achievable rate in the direct channels from BS to WSs.

One main challenge confronting formulation of RRM algorithms as presented in [17] and [18], is that they fall in the class of integer programming problems that require prohibitively exhaustive solution search, with complexity of 
$O\left((M K)^{N}\right)$ [18]. The complexity challenge is primarily due to the OFDMA and the nature of the optimization constraints. The constraints above can be interpreted as a subcarrier can only be assigned to one transmitter-receiver pair, meaning that there is no sharing or intra-cell subcarrier reuse. The optimization problem is revisited in Section VI.

Radio resource management algorithms are sometimes classified according to their output objectives or processing requirements. In terms of their output, the algorithm can be classified as network-centric, achieving high capacity from the perspective of the service provider, or user-centric, providing reliable service and fair share of resources to the WS, or having flavours of both. On the other hand, considering the processing requirements, the classifications are 1) centralized, where the algorithm requires global or centralized knowledge of all interference and channel state information (CSI) for all nodes (WSs, relays) in the network, or 2) semi-distributed, where the algorithm requires a limited global knowledge of network link conditions or 3) fully distributed where the algorithm provides each resource-allocating node the ability to allocate resources based on local channel conditions. While the centralized schemes constitute the bulk of RRM literature, the high signaling overhead (which consumes part of the system throughput) and complexity make them less attractive for OFDMA-based relay networks; therefore, distributed RRM algorithms are expected to attract attention for these type of networks. We also stress that centralized algorithms will continue to occupy an important position since they provide the benchmark performance for assessing the effectiveness of distributed algorithms. In addition, hybrid centralized and distributed schemes may have a place in future network deployment as proposed in [19]. We now briefly discuss some of the basic tools employed for designing RRM solutions.

\section{Scheduling}

Different scheduling policies are available for the selection of WSs to be served based on different network criteria. The most common scheduling algorithms are the following: 1) Round-robin scheduler: This user-centric and fairnessconscious scheduler assigns the same amount of physical resources to WSs in turn. Conventional round-robin scheduler, probably the most natural form of fair scheduling, does not guarantee quality of service since, it neither utilizes the queue state nor exploits the channel variability in the scheduling policy, thereby sacrificing the inherent multiuser diversity and achievable network capacity. 2) Max-SINR scheduler: This network-centric schedulers is the best in terms of total capacity maximization at the price of fairness, as it fully exploits multiuser diversity inherent in the network. 3) Proportional-fair scheduler: This scheduler provides an intermediate solution that realizes the multiuser diversity gains while maintaining fairness across WSs. At any transmit node, this scheduler allocates a subchannel to the WS that maximizes the ratio of its achievable rate on that subchannel to its exponentially weighted average rate. It is observed in [20] that proportional fair scheduling does not guarantee queue stability even for low traffic loads.

The fair throughput and early-deadline-first schedulers are also discussed in many publications in the literature, includ- ing [21], [22], and [23]. The early-deadline-first scheduler is particularly suitable for real-time applications since it can support or allow priority service. Unless modified, the scheduling techniques mentioned above may not deliver optimum performance in the future OFDMA-based relay networks that have many optimization parameters [24]. This fact is recognized in one variant of the round-robin scheduler known as the exhaustive round-robin scheduler, where bursts of un-equal resources are assigned to WSs. The scheduler continues to serve a WS until its queue is exhausted. In this way the overhead requirement is reduced while fairness is sacrificed for increased network capacity.

\section{Routing}

In relay enhanced wireless networks, scheduling and routing resource management decisions should not be isolated. Routing can be viewed as the process of establishing efficient connectivity between nodes over multi-hop links. In communication systems with large number of nodes, there will be diverse link qualities associated with the various links along any route. Therefore, different routing schemes are expected to produce different performance results and overhead [25]. Performing routing and scheduling jointly is known to produce superior performance result compared to decoupled scheduling and routing. A review of some routing metrics and algorithms is available in [26].

\section{Link Adaptation: Adaptive Modulation and Coding}

The best modulation level to employ for transmission can be obtained by adapting modulation according to the instantaneous SNR to achieve the maximum possible transmission rate for a particular target bit error rate (BER); this process is known as adaptive modulation. The combined use of adaptive modulation and channel coding techniques has given rise to what is commonly known as adaptive modulation and coding (AMC). The SNR can be partitioned into a number of consecutive non-overlapping intervals with boundary points obtained by re-arranging the rate expression [27]

$$
r_{i, j, n}=W \log _{2}\left(1+\frac{-1.5 \beta_{i, j, n}}{\ln (5 \mathrm{BER})}\right),
$$

where $r_{i, j, n}$ is the achievable rate, $\beta_{i, j, n}$ is the received SINR from source $i$ at destination $j$ on subchannel $n$ and $W$ is the OFDM subchannel bandwidth. Evaluating the number of bits (spectral efficiency) to employ for the OFDM symbol is sometimes referred to as bit loading. It is important to mention that although adaptive modulation and coding provides more efficient radio resource utilization, it also adds to the overhead signaling since it depends on the knowledge of the CSI at the transmitter.

\section{Cooperative Relaying in OFDMA Networks}

The benefits of cooperative communication for addressing physical layer problems have been well investigated. It is only recently that the cross-layer interaction of cooperative schemes has started to receive attention for the emerging OFDMAbased networks. In fact, cross-layer optimization is identified as an important strategy to ensure overall system performance in wireless networks [28]. The authors in [29] and [30] 
introduce cross-layer optimization to the resource allocation in OFDMA-based relay networks by incorporating a physical layer cooperative technique into the RRM problem formulation. Their treatments are similar in many respects, except that [30] considers multi-hop scenario (more than two hops) while [29] treats only a two-hop case. Furthermore, [30] considers multi-modal relaying (system selects between amplifyand-forward or decode-and-forward relaying, depending on the channel conditions and power allocation), while [29] uses only decode-and-forward relaying. While [29] maximizes the minimum throughput among all BSs under routing and PHY constraints, [30] maximizes network sum utility by optimally choosing the active data stream and allocating power in each tone in conjunction with selecting the best relay node and the best relaying method.

The schemes described in [29] and [30] need to operate in a slow-fading environment to be able to acquire the CSI for implementing the adaptive power and bit-loading in a centralized manner. For this reason, the receiver needs to reliably estimate channel and convey it to the BS. The authors also assume that the OFDM frames are synchronized throughout the network to enable nodes' cooperation at the level of OFDM subcarrier [30]. In an effort to reduce the computational complexity, some conditions are imposed such as the source-relay, source-destination, and relay-destination links all use the same subcarrier. This restriction results in sacrificing part of the inherent frequency diversity in OFDMA systems. Furthermore, the challenges in ensuring such a network-wide synchronization have not been addressed, and the impact on the proposed system is not discussed. Given that OFDM/OFDMA techniques are very sensitive to frequency and timing synchronization errors [31], such an assumption might be too stringent a condition. The impact of these errors on the system performance cannot be left to speculation. Therefore, we believe that further investigation of these issues is required. The performance degradation could result from the loss of orthogonality among subcarriers leading to interference (caused by the inaccurate compensation of frequency offset) or inter-symbol interference due to timing error. Thus, a sufficiently long cyclic prefix or guard interval between adjacent OFDMA symbols may be required to provide protection against these errors. This additional overhead has the effect of reducing the achievable network throughput.

To further emphasize the need for cooperative protocols in the future wireless standards, the IEEE 802.16m working group is considering protocols to enable cooperative communication that is cross-layer optimized. For example, [32] proposes a functional block called cooperative transmission management unit on the upper medium access control. Their description stresses the interaction between the cooperative transmission management unit, scheduling and resource allocator, and the physical layer control, such as interference management, ranging, and link adaptation.

\section{EARLIER RRM RESEARCH IN RELAY NETWORKS}

The need to efficiently utilize the scarce radio spectrum has driven the research trend from fixed reuse patterns to dynamic and very aggressive resource allocation. Even in a non-OFDMA air-interface, the existence of relays presents some challenges in RRM design. Therefore, in the following section we present some of the previous RRM schemes that constitute the building blocks of the discussions in the rest of this survey paper. We start with basic, fixed resource allocation schemes in multicellular networks.

\section{A. Relaying in downlink multicellular networks}

To a large extent, static resource allocation is the common practice in the literature. In particular [33] investigates a static resource allocation in a non-CDMA multi-cell network with 6 fixed relays in each cell. In conjunction with this static allocation, a "pre-configured" relaying channel selection algorithm is used to reduce interference. This algorithm has built-in channel reuse. The reuse is performed in a controlled manner to prevent co-channel interference from increasing to unacceptable levels. This means that a relay is not allowed to reuse any channel in the same cell, but it could reuse the channel from the cell farthest from it (because a cell farthest from a relay most probably has the least co-channel interference to this relay transmission). Note that no channel is reserved for exclusive use of relays, whenever the need arises for relaying, a relay channel is selected from any adjacent cell in a way that keeps the interference within the acceptable level. Different design and path selection criteria are discussed in [25], [33], and [34] in different flavors and forms. Since no channel (frequency) is assigned to relays a priori in these algorithms, the benefits of relaying are achieved without sacrificing system bandwidth.

A centralized downlink joint scheduling and routing in a single cell CDMA EV-DO (EVolution-Data Optimized) system is proposed in [35]. Fairness among WSs is ensured through a queue stability strategy. Relaying is done by reusing the same licensed band used in BS-RS links whereas in some of the earlier works, RS-WS links are assigned an unlicensed band such as for IEEE 802.11. The co-channel interference, a form of intra-cell interference, results from concurrent transmissions of different nodes on the same carrier frequency with different pseudo noise (PN) codes. Relays behave like a WS in uplink and feedback to the BS the ratecontrol information, as well as the queue size information for the assigned WS for each time slot. The joint routing and scheduling examines each possible set of simultaneous active transmitters which can be computationally demanding. For each set of active transmitters, interference power can be calculated at each receiving destination of each link in the set of all possible links defined on the former active set. Relays are allowed to transmit to either WSs or to any other inactive relays during the time frame. The joint scheduling and routing algorithm [35] employs a stochastic drift metric that is calculated for candidate links per each set of active transmitters. This metric is proportional to Shannon capacity and to the maximum difference in queue lengths between source node and destination node across each link. The set of transmitting nodes that achieves the highest drift sum, over all its active links, is the optimal set. Implicitly, the WS that has the largest difference in queue lengths over any active link is scheduled on that link.

A reduced-complexity implementation of this algorithm was also introduced in [35]. In this approach, the infeasible com- 
binations of active links per each set of active transmitters are eliminated. The infeasible combinations violate the following constraints:

- A relay cannot transmit and receive simultaneously.

- Any WS cannot receive simultaneously from two or more different nodes.

- Any transmitting node can only transmit to a single node per time frame.

The WS mobility with such a routing and scheduling algorithm also suggests that data buffered in a relay could be lost if not forwarded to the new serving relay.

In [36], a fixed relay-enhanced multi-cell downlink scenario in FDD mode with TDMA is considered, where the fixed relays are located at the centre of each sector of the cell. By virtue of their relative locations (neighbourhood), antenna directivities, and gains, the cells are organized into in-group BSs representing cells that are mutually interfering with each other. The transmission time frame is divided between the BSs in the dominant interferer group and RSs. In order to schedule and route packets such that dominant interference is minimized, the interference management scheme proposed in [36] utilizes inter-cell coordination among the in-group BSs taking co-channel interference into account. WSs and RSs in a cell estimate their CSI of the links from the serving BS and the in-group BSs and RSs and forward them to the serving BS. From there, they are exchanged among the in-group BSs for informed scheduling decisions. The information exchange can be viewed as a command. Thus, a particular BS might receive different commands from two different groups. When this happens, the BS takes the more conservative command, with the aim to neither cause too much interference to the transmissions of other in-group transmissions nor receive too much interference from other in-group BSs. The objective is to maximize the total network throughput with the least interference suppression. The proposed scheme finds a combination of in-group BS transmissions in the first sub-frame, in addition to the transmission parameters for RS-WS transmission in the next sub-frame. The interference problem is addressed by frequency reuse partitioning from the outset. This a priori resource partitioning is sometimes used in OFDMA networks as well. The downside of this strategy is that it restricts the opportunity in the channel that can be exploited through OFDMA tones. It also requires frequency planning, which can be time-consuming and expensive.

\section{B. Relaying in uplink multicellular networks}

In [37], the static allocation proposed in [33] is extended to FDMA/TDMA in FDD mode for multicellular networks. In contrast to the downlink of [33], an uplink scenario is considered. The objective is to maximize the received SIR values at both BSs and each RS in the uplink. This is achieved by a static allocation that attempts to minimize the interference power received from co-channel terminals. In a cluster size of four cells (the reuse partitioning), the total resources (24 pairs of channels, uplink and downlink) are reused in each cluster; six pairs of channels are assigned to each BS. One pair from the 6 channels of other BS is assigned to each of the 6 RS. Figure 2 shows the layout for the channel allocation, where

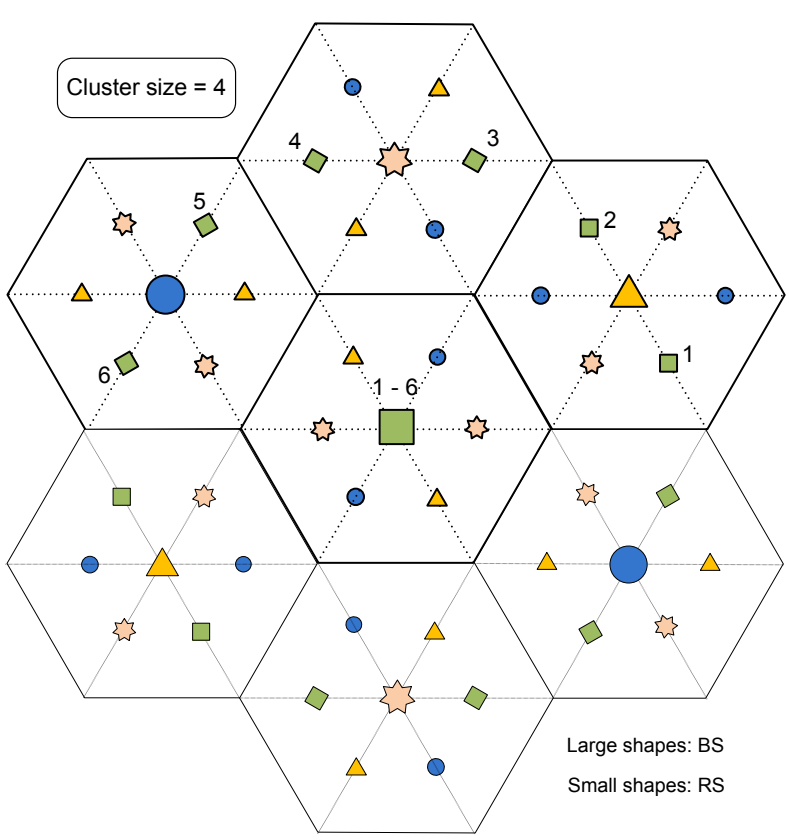

Fig. 2. Static resource allocation in [33] and [37].

nodes (BS or relay) using the same resources are designated with the same shape. The assumed fixed-power allocation calculates the WS's power level to guarantee the minimum required received power from WSs located at cell edges. WSs are assumed to be fixed at the worst interfering positions to the cell under investigation.

This fixed allocation obviously limits the performance gains, due to the suboptimal frequency reuse partitioning. It also sacrifices the inherent multiuser diversity and traffic diversity statistical multiplexing gains.

In a related study to [37], the authors in [38] investigated the performance of an FD/TDMA-based multi-hop fixed cellular network (in which wireless terminals act as relays whenever necessary) with respect to the number of frequency channels. The outage probability, connectivity, and average node throughput are analyzed to explore the dependency of the performance on the number of frequency channels. Results show that implementing multi-hop provides not only significant coverage boost but also high node throughput, as long as there are enough frequency channels. It is observed, on the other hand, that if the network has a limited number of frequency channels, no performance gain can be achieved particularly at high loading values.

In [39], a centralized uplink Universal Mobile Telecommunications System (UMTS) cellular network in FDD mode with Wideband-Code Division Multiple Access (W-CDMA) air interface is considered. The scheme relies on integrated radio resource allocation, where routing is coupled with scheduling. In this scheme, WSs use the same carrier frequency to transmit to either the BS or a RS while the RS uses two different carrier frequencies on the two hops. The proposed algorithm is composed of three entities as shown in Fig. 3: a routing entity, called load based route manager; a resource 


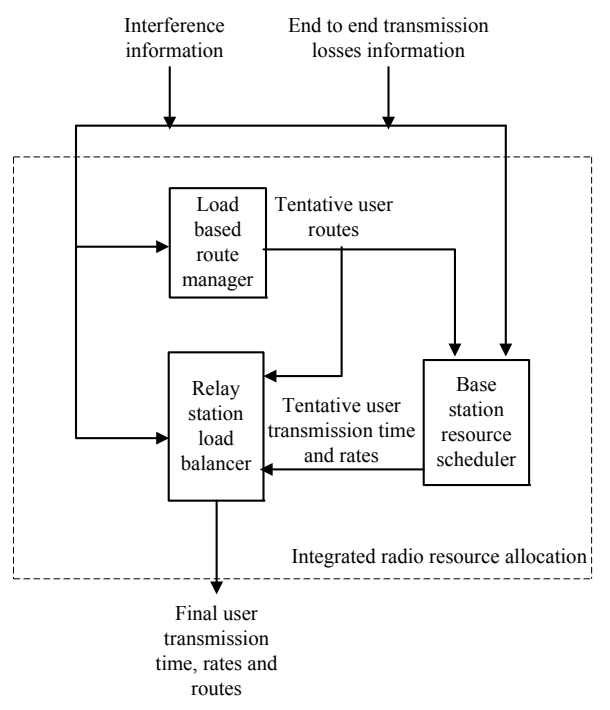

Fig. 3. Structure and sequence of operation of the integrated radio resource allocation in [39].

scheduling entity, called base station resource scheduler; and a refinement entity, called relay station load balancer, that fine-tunes resources assigned to WSs. This refinement process is necessary to prevent overloading the RSs. The proposed scheme uses a path-loss-based benchmark relaying scheme in conjunction with a conventional scheduler. Their optimization cost function, based on system consumed-capacity, is different from the distance and path-loss-based cost functions in [34]. Reference [39] introduces a load-cost indicator, defined as the ratio between the consumed system capacity on a particular route (represented by the interference caused by this WS) to its data rate.

Figure 3 shows the three entities that are fed with the path-loss and interference information. The load-based route manager prepares tentative WS routes based on selection of the routes with least load-cost indicator; then, the base station resource scheduler prepares tentative WS transmission times and rates. The outputs of both entities are input to the relay station load balancer that produces the final WS rates, times and routes such that no relay is overloaded. The relay station load balancer prioritizes WSs by the ratio between the cost of direct-link to that of relayed-link. For some certain system constraints, the throughput can be maximized if every user employs the route with the least load cost indicator among its possible routes. Given that integrating routing to RRM provides superior results, it is observed that integrated radio resource allocation outperforms the reference schemes in several ways.

\section{RRM IN OFDMA FIXED-RELAY NETWORKS}

There is a noticeable similarity between RRM algorithm design for relay-enhanced OFDMA-based networks and earlier RRM algorithms for non-OFDMA relay networks. Thus, the discussions in Section IV (for earlier RRM non-OFDMA relay networks) serve as a link to the literature in RRM in OFDMA relay networks. Essentially, these algorithms are aimed at ensuring prudent utilization of communication resources, and at improving system performance. The following discussions are grouped into centralized and distributed RRM schemes.

\section{A. Centralized RRM in single-cell OFDMA relay networks}

A downlink single-cell network with a single fixed RS is considered in [40], while such a network with multiple fixed RSs is studied in [17]. In both [17] and [40], time-division based half duplex transmission and AMC are assumed. In [40], it is required that WSs feed back their CSI to the BS or the $\mathrm{RS}$ in the form of per-subchannel SNR every time frame. Routing is decoupled from resource allocation to reduce CSI overhead, and an SNR-based path selection algorithm is executed to determine the node to route the data of a WS, an approach that has been previously used for non-OFDMA relay networks [34]. Since the BS-RS subchannels are assumed to have the same average SNR, only the number of required subchannels is estimated for relaying.

In [40] two algorithms (fixed time-division and adaptive time-division) are proposed to improve the cell throughput and coverage while minimizing complexity and overhead requirements. The frame is divided into a BS subframe followed by a RS subframe. In the first fixed time-division algorithm, the $\mathrm{RS}$ performs its own allocation in the RS frame and requests relayed WSs' packets to be forwarded by the BS. The RS, provided it has packets enqueued, allocates the channel to the WS with the highest SNR. Here, throughput is improved from exploiting traffic diversity gains. The set of WSs with high SNR values and no queued packets are expected to be allocated in the next frame. Therefore, the RS requests the WSs' packets to be forwarded. The BS divides its resources between the RS's and the direct WSs' demand. The throughput within the time frame is defined as the average of the BS-RS throughput and the RS-WS throughput.

In the second adaptive time-division algorithm, the allocation is done just as in the first one. However, the time frame is quantized to an integer number of time slots and an iterative time-division adaptation is performed by adding or subtracting a time slot from the BS frame, to maximize the total throughput, which is averaged over the two hops. SNR-based path selection routing is considered, instead of joint routing and scheduling, to reduce the complexity of the algorithm and overhead. Furthermore, the time-division resource partitioning between $\mathrm{BS}$ and $\mathrm{RS}$ transmissions in a frame remains suboptimal despite the gain obtained by optimizing the duration of the subframes.

The objective function in [17] is the total average throughput of both the direct and relayed links presented as a function of SNRs and some indicator (optimization) variables. Two algorithms are proposed to improve the overall cell-throughput while minimizing the system complexity. In both algorithms the BS transmission frame is followed by an equal RS frame. The first algorithm performs subcarrier allocation with a predetermined equal power allocation (the same level for both BS and RS). The second algorithm achieves an optimal joint power and subcarrier allocation. Simulation results show that as the number of RSs increases, the sum rate is increased, while the joint allocation algorithm continues to outperform 
the fixed power allocation algorithm. Without relays, the joint algorithm introduces a marginal gain especially at high SINR regime, and the power allocation part becomes equivalent to water-filling.

In [18], an OFDMA relay network with multiple sources, multiple relays, and a single destination is investigated where resource allocation is considered with fairness (load balancing) constraints on relay nodes. The authors' approach is to transform the integer optimization problem into a linear distribution problem in a directed graph to allow the use of the linear optimal distribution algorithms available in the literature. A (central) decision maker is assumed to know the CSI as well as the role of the node (source/relay/destination). Due to the low speed of nodes, this role designation does not change in an allocation period. The required information is collected at the central unit for allocation decisions. It is also assumed that the subcarriers allocated in the first hop are the same as those in the second hop. This reduction in available frequency diversity gain results in a potential system performance loss. Besides, centralized allocation schemes could sometimes have an unenviable high signaling overhead problems. Thus, in dense relay networks, high complexity and signaling overhead requirements make them unattractive.

In [41] and [42], a multi-hop OFDMA-based downlink system is considered in a single isolated cell, based on a fairness-aware adaptive resource allocation scheme. The CSI is assumed to be available at the BS. The scheme performs adaptive subchannel, path, and power allocation to maximize the system capacity with minimum resources allocated to each WS. The network architecture consists of a BS surrounded by three relays. Dedicated frequency bands are reserved for the BS-RS links, i.e., the resource allocation scheme considers only the links to WSs, either from the BS or RSs. The problem is formulated in a way that a WS can be assigned to either a BS or any of its RSs. However, as a result of excluding the BS-RS links from the resource allocation, a RS is assumed to always have data buffered for all of the potential WSs that can be served by this RS. WSs are allowed to receive allocation signals simultaneously from different nodes and to use the broadcasted resource allocation information to filter out received data.

This formulation leads to a mixed non-linear integer programming optimization problem, which is known to be very difficult to solve in real time. Therefore, a best-effort fairness constraint is imposed to ensure that any WS will be allocated a fixed minimum number of subchannels $\alpha$ where $0<\alpha<$ $N / K$, where $N$ and $K$ are the number of subchannels and WSs, respectively. The parameter $\alpha$ can be tuned such that the optimization problem results in strict resource fairness (as $\alpha \rightarrow N / K$ ) or strict capacity maximization (as $\alpha \rightarrow 0$ ).

The heuristic fairness-aware algorithm is composed of three steps; subchannel allocation, load-balancing, and powerdistribution. The subchannel allocation step is realized in two phases. In the first phase, subchannels are allocated fairly by sorting all the channel gains (over all links and subchannels) in a descending order and allocating subchannels in that order. Once a WS is allocated with its $\alpha$ subchannels the WS is removed from the list. The remaining subchannels are allocated in the second phase to their respective best WSs to improve the capacity in a manner to achieve load-balancing which is to ensure that all nodes are allocated about the same number of subchannels based on the believe that the offered load at any node is proportional to the number of its assigned subchannels. The BS runs the load-balancing algorithm until the difference in the number of allocated subchannels for any two nodes is less than a chosen threshold, or when the difference in the channel gains assigned to different nodes on a selected subchannel is larger than another threshold. These two thresholds can be used to control the number of iterations. In the last step, the BS and RSs distribute the total transmission power equally to the allocated subchannels.

The performance of the capacity maximization heuristic algorithm is close to that of the optimal solution, and significantly outperforms the non-adaptive FDMA resource allocation scheme. In addition, at the price of loss in capacity, the fairness-constrained heuristic algorithm significantly increases the fairness index $F(t)$, based on Jain's index [43], defined as

$$
F(t)=\frac{\left(\sum_{i=1}^{K} r_{i}(t)\right)^{2}}{K \sum_{i=1}^{K} r_{i}^{2}(t)},
$$

where $r_{i}(t)$ 's are WS instantaneous rates. More elaborate discussion on Jain's fairness index is contained in Section VII.

To avoid a difficult non-convex optimization problem, [44] formulates a convex problem in a downlink single-cell scenario by using preassigned subchannels and optimizing only the power allocation. Thereafter, a heuristic scheme for subchannel-allocation using Lagrange dual-decomposition method is adopted. The authors propose a modified waterfilling algorithm that can be solved by an inner-outer bisection method. In the half-duplex relaying scheme, the total transmission time frame is divided equally into two consecutive subframes, BS subframe and RS subframe. In the BS subframe, the BS transmits directly to the single-hop WSs on the set of subchannels $B_{0}$ and transmits to each of the $M$ fixed relays on some other disjoint subchannel sets: $B_{1}, B_{2}, \ldots, B_{M}$. In the RS subframe, RSs transmit to their respective WSs on the disjoint subchannel sets: $S_{1}, S_{2}, \ldots, S_{M}$. The power constraint is applied to the problem in the form of total cell power constraint.

The optimal power allocation algorithm utilizes the Lagrange dual-decomposition instead of a subgradient method, which requires a large number of iterations to converge to the optimal solution. The optimal solution that satisfies KarushKuhn-Tucker (KKT) conditions implies that for any $\mathrm{RS}_{j}$ the sum-rate of the first hop over the set $B_{j}, j \neq 0$, and the sumrate of the second hop over the set $S_{j}$ must be equal. The solution finds a water-filling level $k_{1}$ for all the subchannels in the BS set $B_{0}$ and a pair of filling levels $\left(k_{j 2}\right.$ and $\left.k_{j 3}\right)$ for each $\mathrm{RS}_{j}$, corresponding to the subchannels in the first and second hop sets, $B_{j}$ and $S_{j}$, respectively. The following relation holds for all $j$ :

$$
k_{1}=k_{j 2}+k_{j 3} .
$$

The proposed modified water-filling algorithm employs an inner-outer bisection method, where the outer iterations determine an upper bound of $k_{1}$ by applying the conventional 
water-filling on the subchannels in $B_{0}$, while the inner iterations adjusts $\left\{k_{j 2}\right\}$ and $\left\{k_{j 3}\right\}$ to achieve the equality of the sum-rates of the two hops of all RSs. This equalization is realized in the three-step heuristic algorithm as follows:

Step 1: Generate the modified inverse subchannel SNRs for all $\left\{S_{j}\right\}$, i.e., $1 / \gamma_{1}+1 / \gamma_{2}$ where $\gamma_{1}$ and $\gamma_{2}$ are the normalized SNRs on the first and second hops, respectively.

Step 2: Determine $k_{1}$ using conventional water-filling on the set $B_{0}$ and the modified subchannels in $\left\{S_{j}\right\}$ under the total cell power constraint.

Step 3: Calculate the water-filling power required on the subchannels in $\left\{B_{j}\right\}$ using a bisection method.

The heuristic subchannel-allocation reads thus: the WS with the highest SNR occupies subchannels in $B_{0}$, and the WS with the lowest modified inverse subchannel SNR occupies subchannels in $\left\{S_{j}\right\}$, whereas for $\left\{B_{j}\right\}$ the worst $N_{j}$ subchannels of the BS are chosen.

The inference from this study can be summarized as follows: First, the subchannel allocation is a critical factor in the system performance which requires further investigations for finding an optimal scheme. Second, multi-hop relaying, along with the proposed heuristic subchannel allocation outperforms the optimal resource allocation in OFDMA networks without relays. The proposed optimal solution and the heuristic scheme have not efficiently exploited the inherent multiuser diversity. In addition, the schemes overlook the routing and scheduling in the optimization process. Because the treatment is based on simple network model, there are no provisions for how such schemes can be applied to OFDMA-based multicellular networks. This last point is applicable to many of the current literature, only a handful of papers are available in the open literature investigating relay-enhanced, OFDMA-based air interface technology in multi-cellular networks.

\section{B. Centralized RRM in multi-cell OFDMA relay networks}

In [45], a centralized downlink OFDMA scenario in a multicellular network enhanced with six fixed relays per cell is considered. The proposed scheme considers efficient use of subcarriers via opportunistic spatial reuse within the same cell, as shown in Fig. 4 (i.e., a set of subcarriers used in a BS RS link $\left(S_{2}\right)$ can be reused after 180 degrees angular spacing in a RS - WS link), even when no directional antennas are employed. The data of WSs can be routed through any of the six relays, based on the maximum power received rather than the SINR. This means that the relay selection strategy is insensitive to the amount of interference received. The work in [45] is probably one of the first papers to consider such spatial reuse in a multicell environment, although, with largely oversimplified model. Fading has not been considered except independent lognormal shadowing on links, which means that subcarriers are similar on any particular link. Consequently, the problem was formulated as the minimum number of required subcarriers to satisfy a WS's QoS. This is used in the transmit scheme selection algorithm (TSSA) that switches among single-hop (SH), multi-hop (MH) and $\mathrm{MH}$ with spatial reuse (MHSR).

In TSSA, the only scheme allowed in the interior hexagon is the $\mathrm{SH}$ directly from the $\mathrm{BS}$, whereas outside this region (relay region), TSSA could choose between MH scheme or MHSR that requires less number of subcarriers to satisfy the QoS requirements. With this strategy, an integer programming optimization problem is formulated to maximize the number of WSs with satisfied QoS requirements.

Using the percentage of unsupported WSs as a performance metric, the performances of TSSA and three other preset combinations represented as inner/outer region; $\mathrm{SH} / \mathrm{MH}$, $\mathrm{SH} / \mathrm{MHSR}$ and $\mathrm{SH} / \mathrm{SH}$ are compared. It is observed that a significant increase in the number of supported WSs is achieved when applying TSSA while the other three combinations performed in the following order: $\mathrm{SH} / \mathrm{MH}, \mathrm{SH} / \mathrm{MHSR}$ and $\mathrm{SH} / \mathrm{SH}$. Restricting a cell region to a particular transmission scheme, regardless of the channel conditions, is suboptimal and potentially reduces the performance gains. In addition, no technique was employed to mitigate the co-channel interference. By ignoring channel variations and traffic diversity opportunities, the proposed scheme has not exploited the inherent multiuser and frequency diversity gains.

The idea of grouping WSs based on their locations for allocation purposes appears in [46] in the downlink OFDMAbased multicellular relay-assisted network with global frequency reuse. However, routing is decoupled from resource allocation. In the distance-based relay selection strategy, WSs within a predefined neighbourhood of the BS are restricted to a single-hop transmission from the serving BS. These WSs (users) are called "near-users", while the "far-users" are those that can only receive in two-hop transmissions through the closest RS. Two different time allocation policies are considered in the cell capacity formulations. The first policy is the time-orthogonal policy, in which there is no frequency reuse at all on the three links; the BS-RS backhaul link, the RS-far user link, and the BS-near user link. These links are activated for only a fraction of the total time, $\mu_{b}, \mu_{f}$, and $\mu_{n}$, respectively. In the second policy, on a common subchannel, the BS-RS backhaul link is active within $\mu_{b}$ of the total transmission time, while the BS-near user and RSfar user links are active simultaneously during the remaining $\mu_{n}+\mu_{f}$ portion. Intra-cell interference could occur during the latter concurrent transmissions, as no coordination is employed between the BS and the RS.

Of particular importance is the spectrum reuse capability in this scheme. The RS-far subchannel can be reused in the same cell by introducing a relay reuse factor such that all or a subset of the $M$ RSs in a cell transmit in the time and frequency resource allocated to the BS-near user or each RS has to transmit on a subchannel orthogonal to the subchannels of the other RSs. In the latter case, there is no intra-cell reuse rather bandwidth is partitioned into $M$ orthogonal blocks. In addition to multiuser diversity gains, further capacity improvement is realized by the spectrum reuse among the BS and RSs. It is observed that the performance of the proposed schemes is insensitive to the relay reuse factor, which means that such reuse does not improve the total capacity. The paper concludes that multi-hop relaying represents a viable method for improving the QoS for the far users.

It might have been obvious that in the future wireless networks, centralized RRM schemes are not the best option, considering latency, overhead, system and computational 


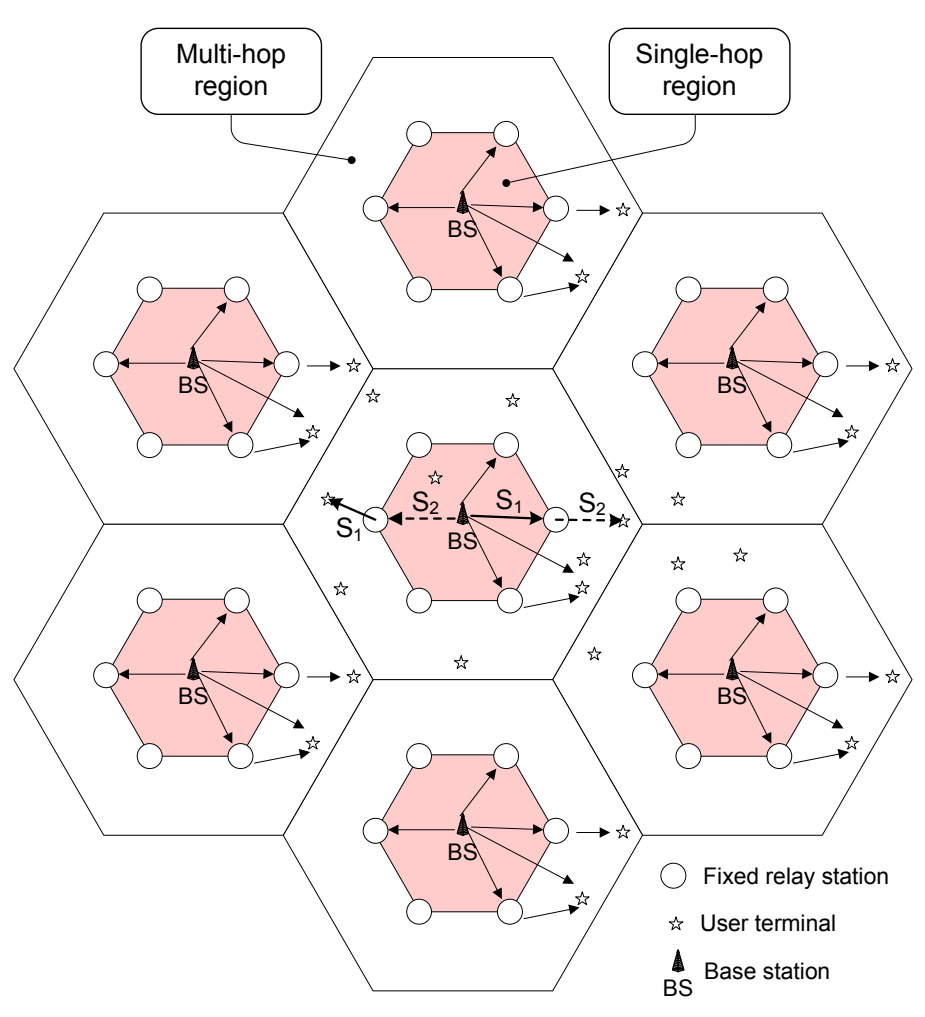

Fig. 4. Network layout and spatial reuse pattern [45].

complexity, among other issues. Hence, the importance of distributed schemes has been recognized. However, there has not been much progress in this front.

\section{Distributed RRM in OFDMA relay networks}

In [47], a "semi-distributed" downlink OFDMA scheme in a single cell enhanced by $M$ half-duplex fixed relays is considered. The scheme divides the WSs into disjoint sets located in the neighbourhoods of the BS and RSs, the approach that is common in the literature and discussed above. The WSs attached to the BS and relays are referred to as BS-WS cluster and RS-WS clusters, respectively. The BS allocates some resources to BS-WS cluster directly and to RS-WS cluster through the RS. Implicitly, it is assumed that all routes have been established prior to resource allocation, regardless of the channel conditions, and that the same subcarrier is used on the two hops, BS-RS and RS-WS. In addition, it is assumed that a protocol is available for gathering CSI and the allocation decisions are broadcasted on a separate control channels.

The authors in [47] classified their algorithms into separate and sequential allocation (SSA) and separate and reuse allocation (SRA). The starting point of these two-step of resource allocation schemes is basically the same. In this first step, each RS, along with its WS cluster, is treated as a large-sized WS with a required minimum rate equal to the sum of all the minimum required rates of the WSs in its cluster. The CSI, in terms of received SNR, is fed back from the RS in the form of two (1-by- $N$ ) vectors carrying the received SNRs at the RS itself and a processed version of the received SNRs at its connected WSs, on $N$ subcarriers. The processing function can result in the maximum SNR, the minimum SNR or the average SNR on each subcarrier. Hence, the BS allocates the resources among its own WSs and these virtual large-sized WSs. In the second step, the RS allocates resources to the WSs in its cluster based on one of two allocation schemes:

- Resources assigned in the first step to that BS-RS link are allocated among the connected WSs, this is the SSA.

- The RS re-allocates all the $N$ subcarriers to its connected WSs regardless of the BS assignments, this is the SRA.

Figure 5 shows the difference in relaying between SSA and SRA within two equal and consecutive time subframes. Since both RS and BS could assign the same subcarriers to their respective WSs, SRA is prone to intra-cell interference

The SSA and SRA are compared with the centralized resource allocation scheme. In the three schemes the best WS on any subcarrier is assigned at any decision-making node, a continuous rate adaptive modulation is employed as in (2). Simulation results for a single cell with one relay show that the semi-distributed scheme, SSA in particular, has a comparable capacity and outage probability performance to the centralized scheme. The SSA shows significant performance stability over the SRA. Intra-cell interference that could occur during the RS subframe in the SRA scheme brings considerable increase in outage probability. The allocation at the RS spans all the $N$ subcarriers and has no coordination at all with that of the BS. Thus, the system could suffer from instability.

In general, the proposed semi-distributed schemes reduce the amount of overhead required to feed back the CSI and 

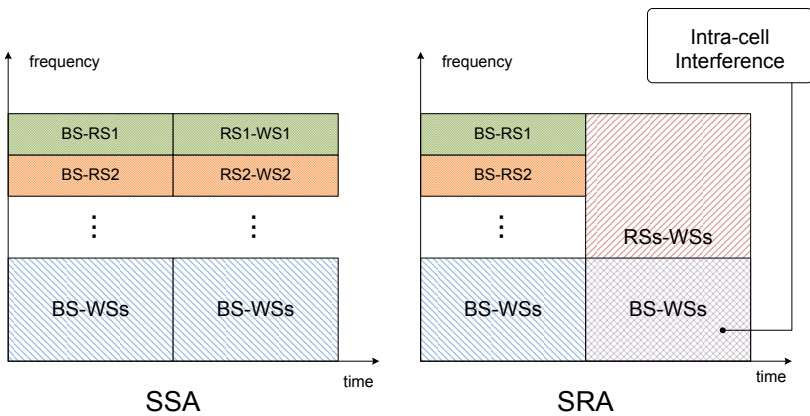

Fig. 5. Half duplex relaying patterns for SSA and SRA as in [47].

minimum rates to the $\mathrm{BS}$, but in the case of SRA, we observed that there is no need for communicating such information to the BS. These schemes fail to exploit the interference avoidance and traffic diversity gains. In addition, there is an inherent loss in performance due to the decoupling of routing and scheduling processes. An excellent tutorial outlining the promises in well-designed distributed RRM schemes is available in [48], except that it does not cover relaying.

\section{Optimization TeChNiques AND COMPleXity ISSUES}

Many approaches are usually adopted in the literature to alleviate the complexity of the RRM schemes. Beside relaxing the conditions on the variables and simplifying the models, some intelligent transformations are often required. For instance, as discussed in Section III, the optimization problem formulated in [18] is NP-hard. Therefore, the prohibitive complexity challenges the feasibility of any optimal algorithm in practical systems. A smart approach that can be used to reduce complexity is to transform the optimization problem to a linear optimal distribution problem in a directed graph. This can subsequently be solved using the known algorithms for linear optimal distribution to obtain the optimal solution with greatly reduced complexity.

In the RRM formulation with cooperative relaying in [29] and [30], a mixed integer programming problem arises. However, when there are sufficiently large number of OFDM tones (e.g., many narrow subcarriers) in the system, the optimal solution of the mixed integer programming has a zero duality gap. The implication of large number of tones is that in multipath channels, there would be many tones that would experience similar channel gains with the neighbouring tones [29] with the effect of time sharing in the frequency domain. The zero duality gap suggests that convex optimization technique can be invoked in solving the mixed-integer programming. With this in mind, a layered approach was employed to solve the optimization problem through the dual method [29]. The Lagrangian multiplying vector which relaxes the achievable rate constraints was also employed. The layered-problem reformulation results in a dual function that depends on application-layer variable (rates) and physical layer variables (e.g., power, bandwidth, and relay-node); each layer, treated as a subproblem, can be individually optimized. The optimal
Lagrange factor can be obtained using the subgradient [30] or ellipsoid method [29]. The complexity of the subgradient updates is polynomial in the dimension of the dual problem, which is the number of potential relays.

The optimization problem formulation in [17] falls in the class of highly complex integer programming problems. The common approach is to relax the binary integer constraints of the optimization variables to continuous positive real variables. By observing the behavior of the objective function, a lemma was deduced, which states that when the subcarriers are allocated optimally, the transmitting and receiving sumrates of each relay are equal. The final relaxed problems are convex, which can be easily solved by simplex or interior point methods (these are known standard optimization tools). In the same manner, to reduce the complexity of the problem formulated in [41] and [42], the integer assignment variables are relaxed to real fractions, converting the problem to a nonlinear programming with concave objective function and convex constraints. In this case, solution can be obtained through gradient methods available in several solvers. The relaxation in the assignment variables implies that any subchannel can be shared by different WSs or different links.

Still, to remedy the difficult non-convex problem formulated in [44], the authors use preassigned subchannels and optimizes only for power allocation to obtain a convex problem. Thereafter, a heuristic scheme for subchannel allocation as a byproduct of applying Lagrange dual-decomposition method is adopted. The proposed optimal power-allocation algorithm is a modified water-filling algorithm that can be solved by an inner-outer bisection method.

\section{FAIRNESS METRIC EXAMPLES}

The aim of this section is to direct readers to the commonly used fairness metrics in the literature. It also attempts to provide some interpretations for these metrics, especially, for the Jain's fairness expression in (3).

1) Jain's fairness index [43]; this index, imported from computer science, takes a value between $1 / K$ and unity, where $K$ is the number of WSs (belonging to the same service class or having equal priority). It possesses low sensitivity at high rates, where small variations between WSs can be neglected, and high sensitivity at low rates where similar variations are not negligible.

2) IEEE $802.16 \mathrm{~m}$ fairness index [32]; this is a WS-specific index, as it represents the ratio of a WS's rate to the average of $K$ WSs' rates (same-priority assumption applies). Thus, each WS's rate is mapped to a value between 0 and $K$.

For the above two metrics, CDF plots of the values measured for different opponent algorithms at the appropriate instants (based on the fairness class, e.g., short-term, long-term, or time-average) can be compared while the most fair CDF would be the closest to a step function at unity.

3) The fairness factor in [28]; this factor has been developed to measure fairness in RRM noncooperative static games. It represents the normalized statistical standard deviation of WS's throughput compared with that of 


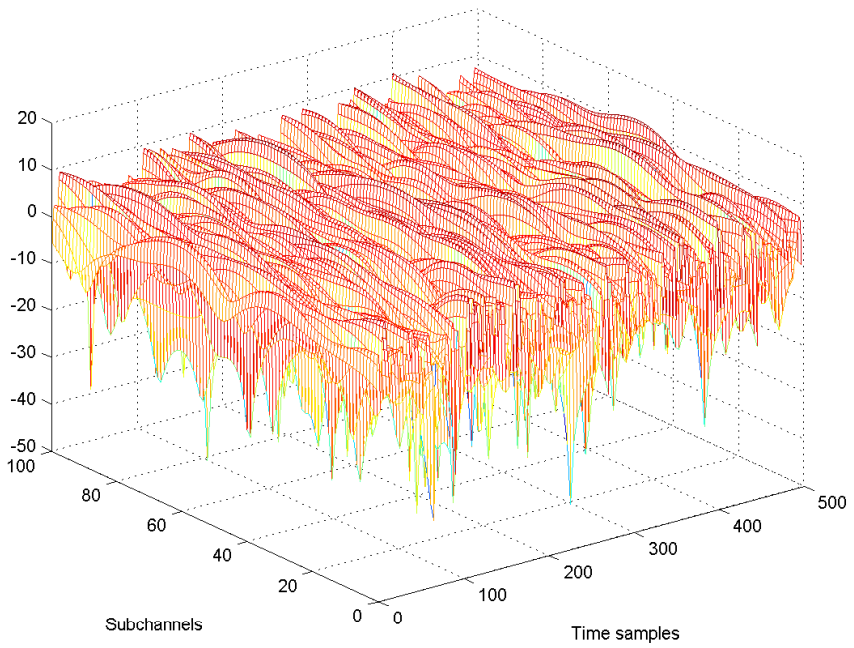

Fig. 6. Time-frequency correlated Rayleigh fading realization for a speed of $v=10 \mathrm{Km} / \mathrm{hr}, f_{c}=2.5 \mathrm{GHz}$.

single-WS case. In contrast to Jain's index, the higher the factor the less the fairness realized among WSs.

\section{ViII. Channel Models And Numerical Examples}

Radio propagation models have significant impacts on the performance of algorithms designed for wireless communication systems. To test new algorithms, the channel models need to be versatile to adequately represent the real-life environments where the systems will be operating. For this reason, many research forums have considered channel models as an important part of their research efforts. The WINNER project invested a significant amount of time in developing spatial channel models that are an evolution of 3GPP's channel model. To capture a wide range of application, the WINNER channel model extends from short range to wide area which covers indoor, typical urban micro-cell, typical urban macrocell, sub-urban macro-cell, rural macro-cell and stationary feeder link and in the frequency range 2 to $6 \mathrm{GHz}$. Frequency diversity and time selectivity are among the features that an RRM scheme could exploit to enhance the performance of the wireless network using OFDM signaling.

\section{A. Simulated channels}

Generating channel samples through IFFT/FFT present a peculiar challenge and it is worth mentioning. The IFFT/FFT approach requires the whole channel samples to be generated and stored prior to simulating the allocation process. Thus, for a large number of subcarriers or subchannels, and potentially large number of links (and/or due to the many fixed relays and nomadic relays), generating large number of independent channel realizations can be computationally exhaustive. We demonstrate the realization of versatile and realistic channel models that incorporate Rayleigh (Fig. 6) and Rician (Fig. 7) fading correlated in time and frequency. Figure 6 shows some realizations of time-frequency correlated Rayleigh fading channel and a 6-tap power delay profile. A

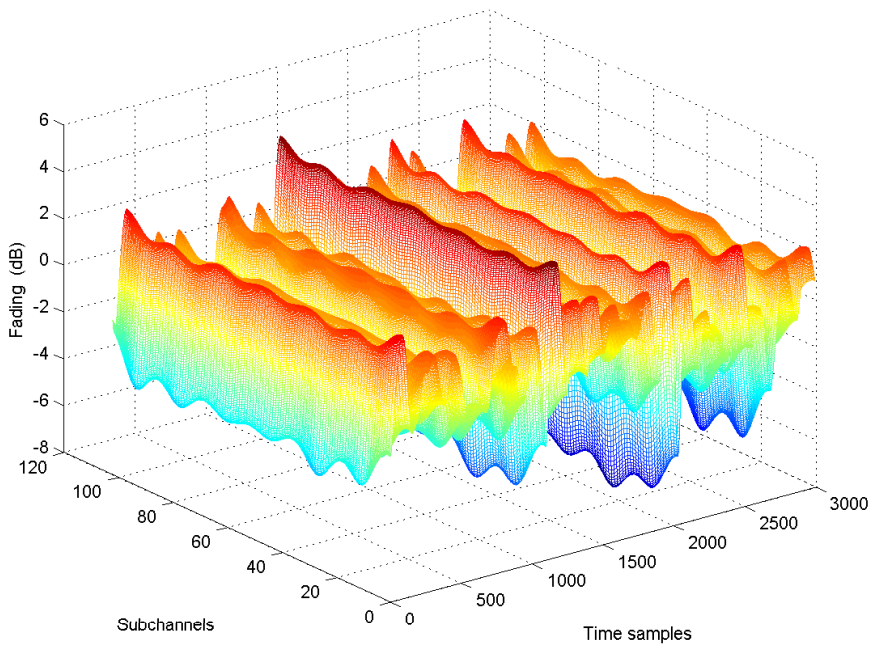

Fig. 7. Time-frequency correlated Rician (K-factor of $10 \mathrm{~dB}$ ) fading realization, 144 subchannels, $f_{d}=4 \mathrm{~Hz}, f_{c}=2.5 \mathrm{GHz}$.

channel model representing a higher Doppler spread and lineof-sight scenario is shown in Fig. 7. In these figures, the relative speed $v(\mathrm{~m} / \mathrm{s})$ between the transmitter and receiver, the operating carrier frequency $f_{c}$ (in $\mathrm{Hz}$ ) are given. Therefore, the maximum Doppler spread $f_{d}$ (in $\mathrm{Hz}$ ) can be calculated using [49]

$$
f_{d}=\frac{v}{c} f_{c}
$$

where $c($ in $\mathrm{m} / \mathrm{s})$ is the speed of light. The delay spread for the simulated channels can also be obtained from the power delay profile, $\mathbf{h}=\left[\begin{array}{llllll}0 & -1 & -9 & -10 & -15 & -20\end{array}\right] \mathrm{dB}$ and tap delays, $\boldsymbol{\tau}=\left[\begin{array}{llllll}0.000 & 0.300 & 0.700 & 1.100 & 1.700 & 2.500\end{array}\right] \mu s$. The essential features of the channel that can be exploited by OFDM technique is obvious in the two figures. The dynamic fading across frequency and time provides the opportunity to use the channel when it is most favorable for transmission.

Despite this opportunity, some of the works in the literature rely only on the frequency diversity, and assume time-invariant channels with little or no mobility [17], [44]. However, in the single-cell relay networks in [40] and [47], time-frequencycorrelated Rayleigh fading channels, with exponential powerdelay profile for WS links using Jake's model, are used.

\section{B. Numerical examples}

Consider an OFDMA-based multicellular relay network with $K=15$ and 25 (number of WS per cell) and $M=3$ and 6 (number of relays per cell). The fairness-aware, RRM algorithm described in [50] is employed as the underlying resource allocation scheme for this network. Since traffic diversity is exploited in their algorithm, [50] assumes independent Poisson processes for WS packet arrival at BS queues. The average arrival rate per WS is 632 packets per second, each packet is 188 bytes. The operating frequency and spectrum are $2.5 \mathrm{GHz}$ and $20 \mathrm{MHz}$, respectively. The performance figures (Fig. 8 through Fig. 11) are given mainly for three reasons: 1) to expose the impact of relays in OFDMA-based relay networks, 2) to expose the importance of efficient or intelligent RRM 


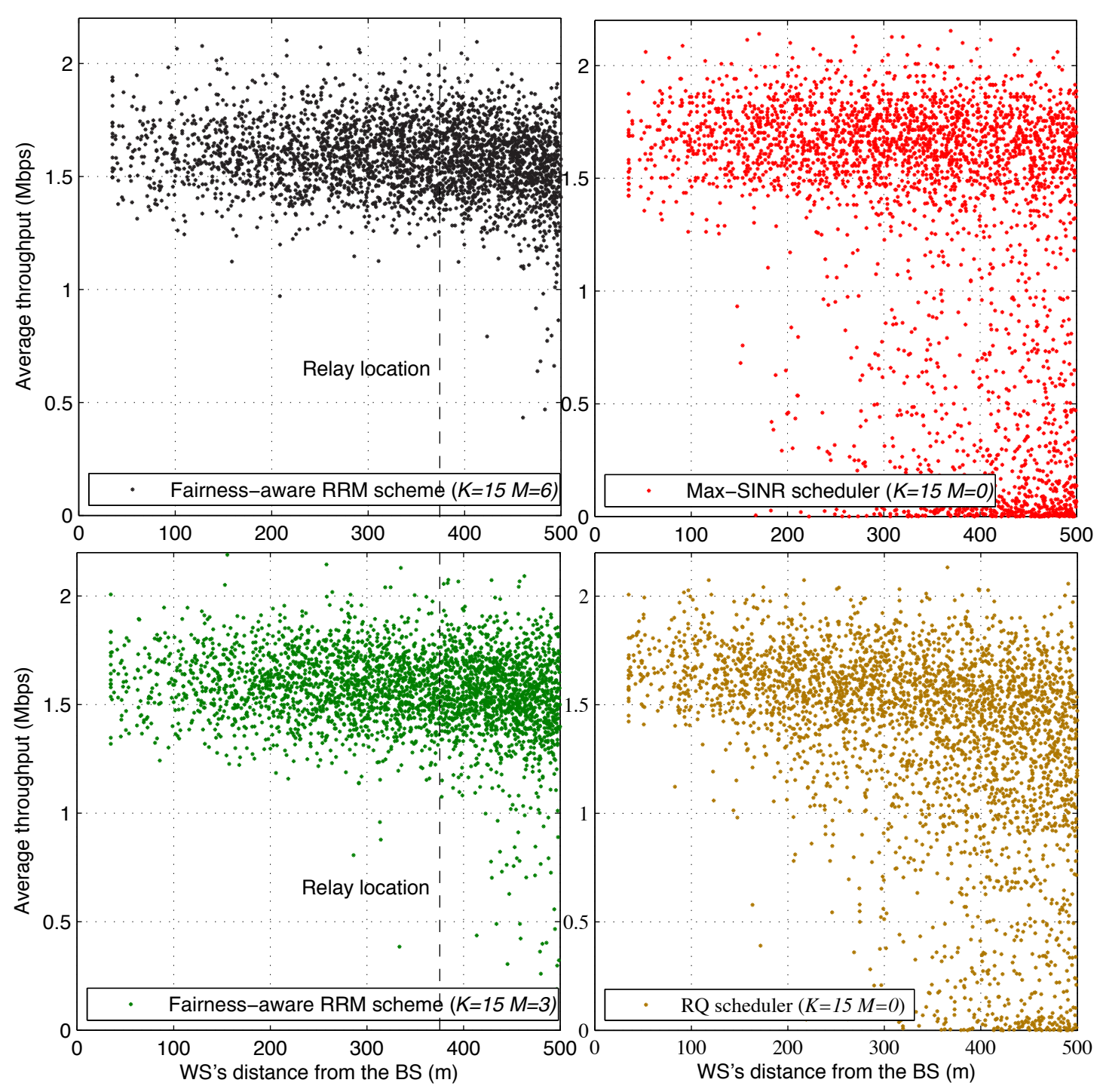

Fig. 8. The scatter plot of WS throughput for a fairness-aware RRM scheme compared to a capacity-greedy scheme. Note that this Max-SINR implementation allocates resources to the best users among only those with traffic enqueued.

schemes in such networks, and 3) to demonstrate how to interpret the fairness metrics discussed in the paper, the Jain's and IEEE $802.16 \mathrm{~m}$.

Figure 8 shows the average throughput as a function of WS distance from the BS using a scatter plot. The plot is obtained by time averaging the throughput for each WS within a drop (100 of them) with fixed location and shadowing. It can be seen (through the almost flat performance from the BS to the cell edge) that there is a uniform average throughput across the cell area compared with the Max-SINR scheduler where some WSs are rate starved (close to zero rates). Thus, with a smart RRM scheme, a fair service and ubiquitous coverage are provided for all WSs regardless of their locations, which is not the case for the capacity greedy or fairness-unaware schedulers such as the Max-SINR scheduler.

The scatter points can be transformed to a form that can facilitate further understanding. The scatter points can be collected, through the CDF plots, to study the statistics of timeaveraged WS throughput as shown in Fig. 9. If we consider a target average throughput of $1.2 \mathrm{Mbps}$, the outage probability is about $1.5 \%$ and $3.0 \%$ for $M=6$ and $M=3$, respectively, as compared to $27 \%$ for the $R \times Q$ scheduler (the fairnessaware algorithm in [50] when relays are not employed) and $38 \%$ for the Max-SINR. The importance of intelligent RRM scheme is quite obvious considering the significant outage reduction. Of particular importance is the information that can be obtained from this plot which is associated with the celledge WSs. The LTE evaluation defines cell-edge WSs as those in the lower tail, $5^{\text {th }}$ percentile in the throughput CDF. From Fig. 9, it is observed that excellent throughput performances are realized, using the $R \times Q$ scheduler and the fairnessaware RRM scheme with $M=3$ and $M=6$. Therefore, the fairness-aware RRM scheme (and $R \times Q$ scheduler) provides a substantial improvement in the $5^{\text {th }}$ percentile performance compared to the Max-SINR scheduler.

The IEEE $802.16 \mathrm{~m}$ index is an important measure of timeaverage fairness behavior based on the time-average WS throughput rates. The interpretation of the index is, the closer 


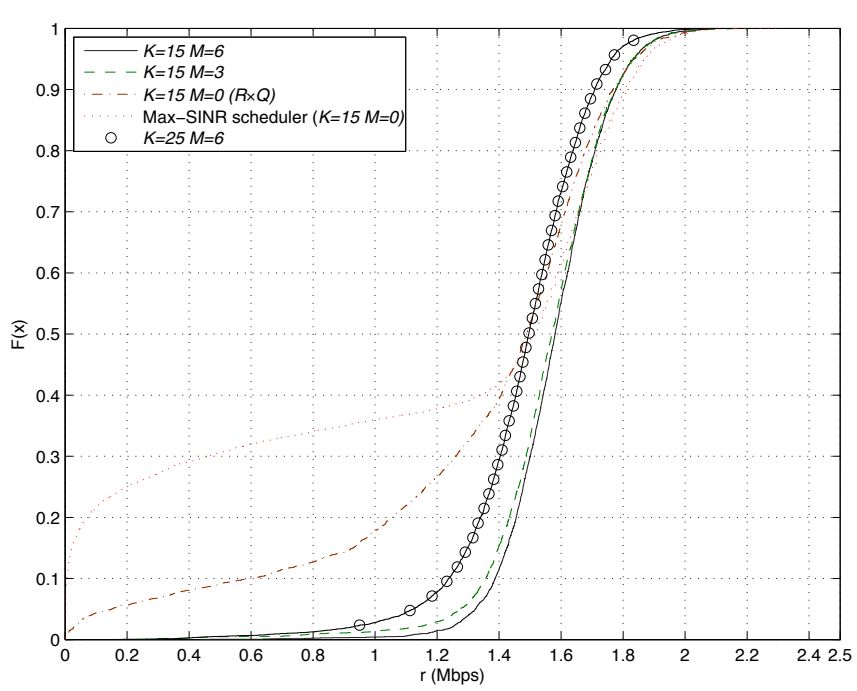

Fig. 9. The time-averaged WS throughput is compared for fairness-aware RRM schemes and a capacity-greedy scheme, for different number of WSs per cell and relays per cell.

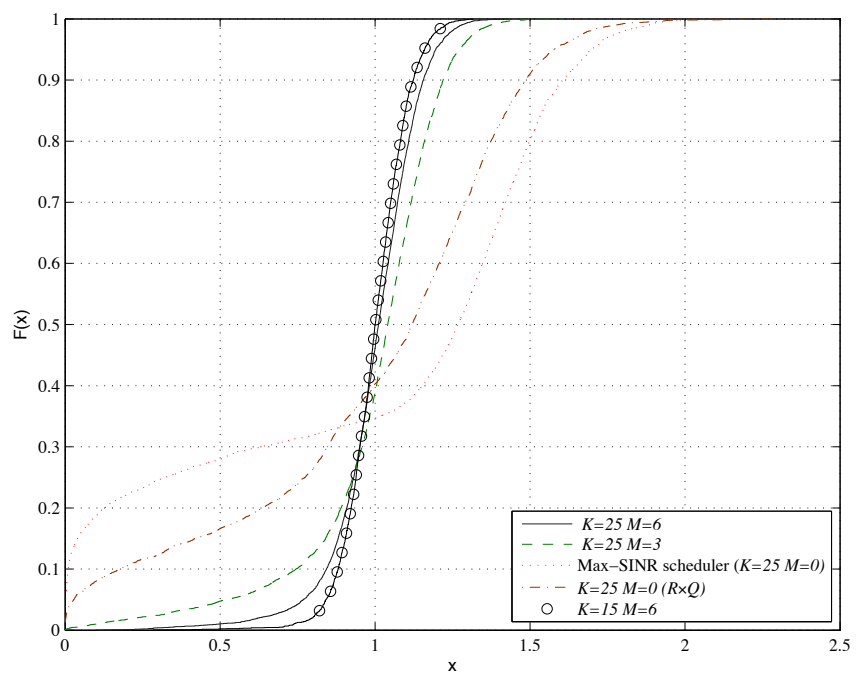

Fig. 10. Fairness behavior using IEEE 802.16m index for a fairness-aware RRM scheme and a capacity-greedy scheme, for different number of WSs per cell and relays per cell.

the CDF plot is to a step function at unity, the more fair the scheme is. As observed in Fig. 10, the fairness-aware RRM scheme provides a superior fair service compared to the MaxSNIR scheduler. In addition, Fig. 11 is provided to explain the Jain's index. While this index is lower-bounded by $1 / K$, it is unity if all the $K$ WSs achieve the same rate. As with IEEE 802.16m index, the closer the CDF to a unit step at unity the more short-term (instantaneous) fairness the scheme achieves. Thus, it is observed in Fig. 11 that with the underlying RRM scheme, more relays can be used to provide more fairness.

\section{CONCLUSION}

This paper provides an overview on RRM schemes in relay-enhanced OFDMA-based wireless networks. Numerous publications have highlighted the need for efficient resource management in OFDMA networks. Although the literature of RRM in OFDMA-based relay networks has begun to draw

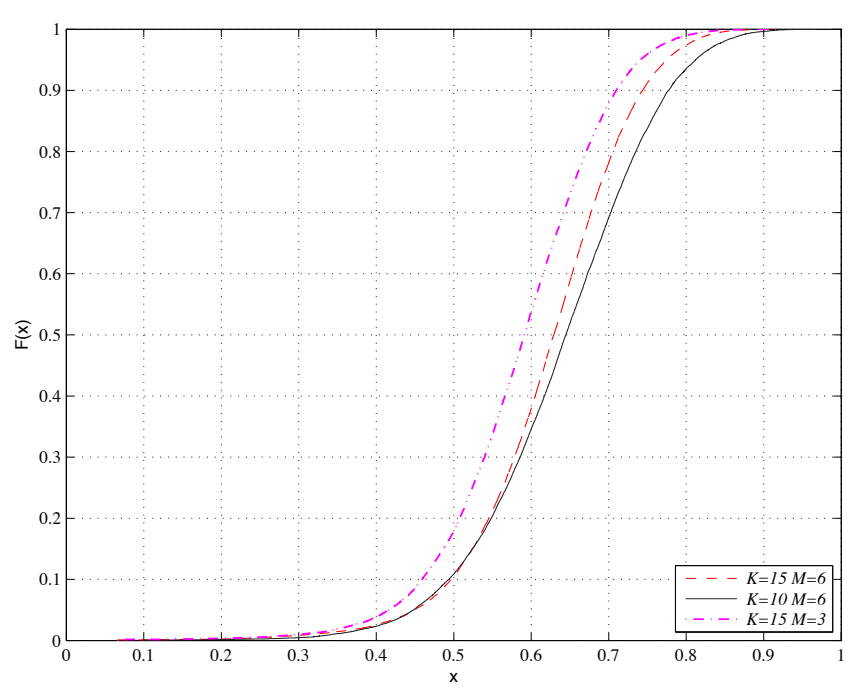

Fig. 11. Jain's fairness behavior of the fairness-aware RRM schemes, for different number of WSs per cell and relays per cell.

attention, only a scant number of papers have investigated OFDMA-based relay networks in multicellular environments. In general, these papers proposed algorithms that exploit the variations in wireless channels by adaptively allocating scarce communication resources to network entities to either maximize or minimize certain network metrics for some given constraints. Some of the proposed algorithms are designed to reduce the required signalling overhead compared to the optimal solution, when the optimal solution involves prohibitive complexity. For instance, there are on-going research efforts towards finding more efficient centralized RRM algorithms, since most of those proposed in the existing literature are overly complex. Furthermore, significant savings in overhead and system complexity can be obtained through distributed resource allocation schemes. Therefore, research in distributed RRM algorithms in OFDMA-based relay networks has started drawing attention. While the literature is steadily growing, a rich list of references has been identified and provided to guide interested readers as they explore this emerging area.

\section{REFERENCES}

[1] H. Yanikomeroglu, "Fixed and mobile relaying technologies for cellular networks," Second Workshop on Applications and Services in Wireless Networks (ASWN'02), pp. 75-81, July 2002.

[2] R. Pabst, B. Walke, D. Schultz, P. Herhold, H. Yanikomeroglu, S. Mukherjee, H. Viswanathan, M. Lott, W. Zirwas, M. Dohler, H. Aghvami, D. Falconer, and G. Fettweis, "Relay-based deployment concepts for wireless and mobile broadband cellular radio," IEEE Communications Magazine, 42(9), pp. 80-89, Sept. 2004.

[3] G. Li and H. Liu, "Downlink dynamic resource allocation for multicell OFDMA systems," IEEE Vehicular Technology Conference, pp. 1698-1702, Oct. 2003.

[4] G. Li and H. Liu, OFDM-based Broadband Wireless Networks: Design and Optimization, Wiley, Oct. 2005.

[5] J. Jang and K. B. Lee, "Transmit power adaptation for multiuser OFDM systems," IEEE Journal on Selected Areas in Communication, 21(2), pp. 171-178, Feb. 2003.

[6] S. Pietrzyk and G. J. M. Janssen, "Radio resource allocation for cellular networks based on OFDMA with QoS guarantees," IEEE Global Telecommunications Conference, Nov. 2004.

[7] D. Niyato and E. Hossain, "Adaptive fair subcarrier/rate allocation in multirate OFDMA networks: Radio link level queuing performance analysis," IEEE Trans. Veh. Technol., 55(6), Nov. 2006. 
[8] S. Sadr, A. Anpalagan, and K. Raahemifar, "Radio resource allocation algorithms for the downlink of multiuser OFDM communication systems," IEEE Commun. Surveys Tutorials, 11(3), pp. 92 - 106, 3rd Quarter 2009.

[9] M. Salem, A. Adinoyi, H. Yanikomeroglu, and D. Falconer, "Opportunities and challenges in OFDMA-based cellular relay networks: A radio resource management perspective," IEEE Trans. Veh. Technol., issue 99, Jan 2010 .

[10] S. Kuan, "Overview of PCS unlicensed wireless standards in the US," IEEE International Symposium on Personal, Indoor and Mobile Radio Communications, pp. 958-962, Oct. 1996.

[11] T. Rouse, I. Band, and S. McLaughlin, "Capacity and power investigation of opportunity driven multiple access (ODMA) networks in TDD-CDMA based systems," IEEE International Conference on Communications, pp. 3202-3206, May 2002.

[12] H. Zhu and G. Cao, "On improving the performance of IEEE 802.11 with relay-enabled PCF," Springer Journal on Mobile Networks and Applications, pp. 423-434, Nov. 2004.

[13] H. Zhu and G. Cao, "rDCF: A relay-enabled medium access control protocol for wireless ad hoc networks," IEEE Trans. Mobile Comput., 5(9), pp. 1201-1214, Sept. 2006.

[14] G. R. Hiertz, S. Max, R. Zhao, D. Denteneer, and L. Berlemann, "Principles of IEEE 802.11s," International Conference on Computer Communications and Networks, pp. 1002-1007, Aug. 2007.

[15] M. Sternad, T. Svensson, T. Ottosson, A. Ahlen, A. Svensson, and A. Brunstrom, "Towards systems beyond 3G based on adaptive OFDMA transmission," Proc. IEEE, 95(12), pp. 2432-2455, Dec. 2007.

[16] IEEE P802.16j/D9, "Draft IEEE standard for local and metropolitan area networks Part 16: Air interface for fixed and mobile broadband wireless access systems: multihop relay specification," Feb. 2009.

[17] W. Nam, W. Chang, S.-Y Chung, and Y. Lee, "Transmit optimization for relay-based cellular OFDMA systems," IEEE International Conference on Communications, pp. 5714-5719, June 2007.

[18] G. Li and H. Liu, "Resource allocation for OFDMA relay networks with fairness constraints," IEEE J. Sel. Areas Commun., 24(11), pp. 2061-2069, Nov. 2006.

[19] E. Tragos, A. Mihovs, E. Mino, J. Lou, R. Fracchia, G. Vivier, and X. Xue, "Hybrid RRM architecture for future wireless networks," IEEE International Symposium on Personal, Indoor and Mobile Radio Communications, pp. 1-5, Sept. 2007.

[20] M. Andrews, "Instability of the proportional fair scheduling algorithm for HDR," IEEE Trans. Wireless Commun., pp. 1422-1426, Sept. 2004.

[21] Y. Ma, "Proportional fair scheduling for downlink OFDMA," IEEE International Conference on Communications, pp. 4843-4848, June 2007.

[22] Z. Shen, J. G. Andrews, and B. L. Evans, "Adaptive resource allocation in multiuser OFDM systems with proportional rate constraints," IEEE Trans. Wireless Commun., pp. 2726- 2737, Nov. 2005.

[23] C. Y. Huang, H.-H Juan, M.-S Lin, and C.-J. Chang, "Radio resource management of heterogeneous services in mobile WIMAX systems," IEEE Wireless Commun., pp. 20-26, Feb. 2007.

[24] B. Can, H. Yanikomeroglu, F. Atay Onat, E. Carvalho, and H. Yomo, "Efficient cooperative diversity schemes and radio resource allocation for IEEE 802.16j," IEEE Wireless Communications and Networking Conference, pp. 36-41, Apr. 2008.

[25] V. Sreng, H. Yanikomeroglu, and D. Falconer, "Capacity enhancement through two-hop relaying in cellular radio systems," IEEE Wireless Communications and Networking Conference, pp. 881-885, Mar. 2002.

[26] Y. Park and E.-S. Jung, "Resource-aware routing algorithms for multihop cellular networks," International Conference on Multimedia and Ubiquitous Engineering, pp. 1164-1167, Apr. 2007.

[27] X. Qiu and K. Chawla, "On the performance of adaptive modulation in cellular systems," IEEE Trans. Commun., 47(6), pp. 884-895, June 1999.

[28] Z. Han and K. J. R. Liu, Resource Allocation for Wireless Networks: Basics, Techniques, and Applications, Cambridge, 2008.

[29] S. Kim, X. Wang, and M. Madihian, "Resource allocation in multi-hop OFDMA wireless backhaul networks with cooperative relaying," IEEE Global Telecommunications Conference, pp. 5205-5209, Nov. 2007.

[30] T. C. $\mathrm{Ng}$ and W. Yu, "Joint optimization of relay strategies and resource allocations in cooperative cellular networks," IEEE J. Sel. Areas Commun., 25(2), pp. 328-339, Feb. 2007.

[31] M. Morelli, C.-C. J. Kuo, and M.-O. Pun, "Synchronization techniques for orthogonal frequency division multiple access (OFDMA): A tutorial review," Proc. IEEE, 95(7), pp. 1394-1427, July 2007.

[32] IEEE C802.16m-07/306, Protocol structure to support cooperative transmission," "IEEE 802.16 Broadband Wireless Access Working Group," http://www.ieee802.org/16/tgm/contrib/C80216m-07_306.pdf.
[33] H. Hu, H. Yanikomeroglu, D. Falconer, and S. Periyalwar, "Range extension without capacity penalty in cellular networks with digital fixed relays," IEEE Global Telecommunications Conference, pp. 3053-3057, Nov. 2004.

[34] V. Sreng, H. Yanikomeroglu, and D. Falconer, "Relayer selection strategies in cellular networks with peer-to-peer relaying,", IEEE Vehicular Technology Conference, pp. 1949-1953, Oct. 2003.

[35] H. Viswanathan and S. Mukherjee, "Performance of cellular networks with relays and centralized scheduling," IEEE Trans. Wireless Commun., 4(5), pp. 2318-2328, Sept. 2005.

[36] M. Rahman and H. Yanikomeroglu, "QoS provisioning in the absence of ARQ in cellular fixed relay networks through inter-cell coordination," IEEE Global Telecommunications Conference, pp. 1-5, Nov. 2006.

[37] P. Li, M. Rong, T. Liu, and D. Yu, "Interference modeling and analysis in two-hop cellular network with fixed relays in FDD mode," International Conference on Wireless Communications, Networking and Mobile Computing, pp. 427-451, Sept. 2005.

[38] M. Ahmed, I. Syed, and H. Yanikomeroglu, "On the performance of TDMA-based multi-hop fixed cellular networks with respect to available frequency channels," IET Communications, 2(9), pp. 1196-1204, Oct. 2008.

[39] Y. Liu, R. Hoshyar, X. Yang, and R. Tafazolli, "On the radio resource allocation in enhanced uplink UTRA-FDD with fixed relay stations," IEEE International Symposium on Personal, Indoor and Mobile Radio Communications, pp. 1611-1615, Sept. 2005.

[40] M. Kaneko and P. Popovski, "Radio resource allocation algorithm for relay-aided cellular OFDMA system," IEEE International Conference on Communications, pp. 4831-4836, June 2007.

[41] C. Bae and D.-H. Cho, "Fairness-aware adaptive resource allocation scheme in multi-hop OFDMA systems," IEEE Commun. Lett., 11(2), pp. 134-136, Feb. 2007.

[42] C. Bae and D.-H. Cho, "Adaptive resource allocation based on channel information in multi-hop OFDM systems," IEEE Vehicular Technology Conference, pp. 1-5, Sept. 2006.

[43] R. Jain, The Art of Computer Systems Performance Analysis: Techniques for Experimental Design, Measurement, Simulation and Modeling, New York: Wiley, 1991.

[44] R. Kwak and J. M. Cioffi, "Resource-allocation for OFDMA multihop relaying downlink systems," IEEE Global Telecommunications Conference, pp. 3225-3229, Nov. 2007.

[45] J. Lee, S. Park, H. Wang, and D. Hong, "QoS-gurarantee transmission scheme selection for OFDMA multi-hop cellular networks," IEEE International Conference on Communications, pp. 4587-4591, June 2007.

[46] Ö. Oyman, "Opportunistic scheduling and spectrum reuse in relaybased cellular OFDMA networks," IEEE Global Telecommunications Conference, pp. 3699-3703, Nov. 2007.

[47] M. Kim and H. Lee, "Radio resource management for a two-hop OFDMA relay system in downlink," IEEE Symposium on Computers and Communications, pp. 25-31, July 2007.

[48] D. Gesbert S. Kiani, A. Gendhemsjo, and G. Oien, "Adaptation, coordination, and distributed resource allocation in interference-limited wireless networks," Proc. IEEE, 95(12), pp. 2393-2409, Dec. 2007.

[49] T. S. Rappaport, Wireless Communications: Principle and Practice, 2nd edition, Prentice Hall, 2001.

[50] M. Salem, A. Adinoyi, M. Rahman, H. Yanikomeroglu, D. Falconer, Y.D. Kim, and E. Kim, "Fairness-aware joint routing and scheduling in OFDMA-based multi-cellular fixed relay networks," IEEE International Communications Conference, June 2009. 


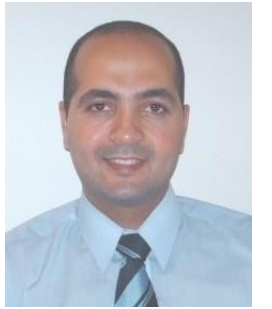

Mohamed Rashad Salem (S'06) received his B.Sc. in Communications and Electronics from the department of Electrical Engineering, Alexandria University, Egypt, in 2000. He was nominated and hired as a faculty member in the department of Engineering Mathematics, Alexandria University, from which he received his M.Sc. degree and was promoted to the position of Assistant Lecturer in Feb 2006. Within this period he has gained wide experience in Research \& Development and collaboration with industrial parties. In June 2006, he has received a scholarship form the department of Systems and Computer Engineering, Carleton University, Ottawa, Ontario, Canada, to pursue his Ph.D. Towards that end, he has been conducting research in collaboration with Samsung Electronics on advanced radio resource management in next generation wireless networks. His research interests encompass stochastic modeling, congestion control, and optimization techniques.

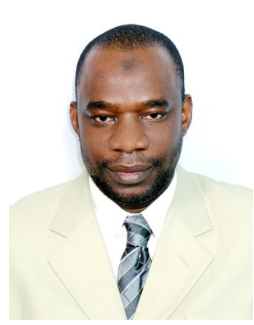

Abdulkareem Adinoyi received a B.Eng degree from the University of Ilorin, Nigeria, in 1992 a M.S degree from the King Fahd University of Petroleum and Minerals (KFUPM), Dhahran, Saudi Arabia, in 1998 and a Ph.D degree from Carleton University, Ottawa, Canada, in 2006. All the degrees are in electrical engineering. From April 1993 to August 1995 Mr. Adinoyi was with Dubi Oil Limited, Port Harcourt, Nigeria as an instrument/electrical engineer. Between September 1995 and October 1998 he was with KFUPM as a research assistant and between January 1999 and August 2002 he held the position of a lecturer at the department of electrical engineering, KFUPM. He is currently a senior research associate at the Department of Systems and Computer Engineering (SCE) at Carleton University. Between January 2004 and December 2006, he participated in the European Union 6th Framework integrated project - the WINNER. From January 2007 to August 2007, he was briefly at Qassim University, Saudi Arabia where he held the position of an assistant professor of electrical and electronic engineering. Between September 2007 and July 2009, he worked, as project technical manager, on Samsung's advanced radio resource management project for OFDMA-based relay-enhanced broadband wireless networks under the auspices of SCE. He is currently at Swedtel Arabia as consultant for Saudi Telecommunication Company. Dr. Adinoyi's current research interests are in infrastructure-based multihop and relay networks, cooperative diversity schemes and protocols, efficient radio resource management techniques for the next-generation wireless networks, and technology evolution.

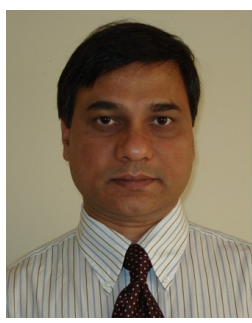

Mahmudur Rahman received his B.Sc. degree in Electrical and Electronic Engineering from Bangladesh University of Engineering and Technology (BUET), Dhaka, Bangladesh, in 1991. He obtained an M. Eng. degree in Telecommunications from Asian Institute of Technology (AIT), Bangkok, Thailand and an M.A.Sc. degree in Electrical Engineering from Carleton University, Ottawa, Canada, in 1994 and 2004, respectively.

He worked for Bangladesh Atomic Energy Commission (Bangladesh), Johnson Electric Industrial Ltd. (Thailand), and ACE Electronics Industries Co., Ltd. (Thailand) at different times of his career. He was involved in the World INitiative NEw Wireless Radio (WINNER) research project from 2004 to 2007. He is currently pursuing a Ph.D. degree in Electrical Engineering at Carleton University and working as a Research Engineer in Communications Research Centre (CRC), Ottawa, Canada. His current research interests include radio resource management, multi-hop wireless networks, inter-cell coordination, interference management, and cognitive radio systems.

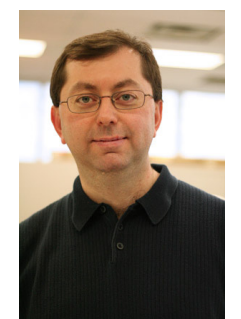

Halim Yanikomeroglu received a B.Sc. degree in Electrical and Electronics Engineering from the Middle East Technical University, Ankara, Turkey, in 1990, and a M.A.Sc. degree in Electrical Engineering (now ECE) and a Ph.D. degree in Electrical and Computer Engineering from the University of Toronto, Canada, in 1992 and 1998, respectively. He was with the R\&D Group of Marconi Kominikasyon A.S., Ankara, Turkey, from 1993 to 1994.

Since 1998 Dr. Yanikomeroglu has been with the Department of Systems and Computer Engineering at Carleton University, Ottawa, where he is now an Associate Professor with tenure. Dr. Yanikomeroglus research interests cover many aspects of the physical, medium access, and networking layers of wireless communications with a special emphasis on multihop/relay/mesh networks and cooperative communications. Dr. Yanikomeroglus research is currently funded by Samsung (SAIT, Korea), Huawei (China), Communications Research Centre of Canada (CRC), Research in Motion, Canada, and NSERC. Dr. Yanikomeroglu is a recipient of the Carleton University Research Achievement Award 2009.

Dr. Yanikomeroglu has been involved in the steering committees and technical program committees of numerous international conferences; he has also given 18 tutorials in such conferences. Dr. Yanikomeroglu is a member of the Steering Committee of the IEEE Wireless Communications and Networking Conference (WCNC), and has been involved in the organization of this conference over the years, including serving as the Technical Program Co-Chair of WCNC 2004 and the Technical Program Chair of WCNC 2008. Dr. Yanikomeroglu is the General Co-Chair of the IEEE Vehicular Technology Conference to be held in Ottawa in September 2010 (VTC2010-Fall).

Dr. Yanikomeroglu was an editor for IEEE Transactions on Wireless Communications [2002-2005] and IEEE Communications Surveys \& Tutorials [2002-2003], and a guest editor for Wiley Journal on Wireless Communications \& Mobile Computing. He was an Officer of IEEE's Technical Committee on Personal Communications (Chair: 2005-06, Vice-Chair: 2003-04, Secretary: 2001-02), and he was also a member of the IEEE Communications Society's Technical Activities Council (2005-06).

Dr. Yanikomeroglu is an Adjunct Professor at the Prince Sultan Advanced Technologies Research Institute, King Saud University, Riyadh, Saudi Arabia. $\mathrm{He}$ is also a registered Professional Engineer in the province of Ontario, Canada.

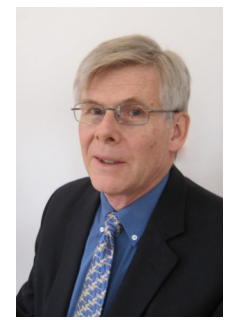

David Falconer received the B.A. Sc. degree in Engineering Physics from the University of Toronto in 1962, the S.M. and Ph.D. degrees in Electrical Engineering from M.I.T. in 1963 and 1967 respectively, and an honorary doctorate of science from the University of Edinburgh in 2009. After a year as a postdoctoral fellow at the Royal Institute of Technology, Stockholm, Sweden he was with Bell Laboratories from 1967 to 1980 as a member of technical staff and group supervisor. During 1976-77 he was a visiting professor at Linköping University, Linköping, Sweden. Since 1980 he has been with Carleton University, Ottawa, Canada, where he is now Professor Emeritus and Distinguished Research Professor in the Department of Systems and Computer Engineering. His current research interests center around beyond-third-generation broadband wireless communications systems. He was Director of Carleton's Broadband Communications and Wireless Systems (BCWS) Centre from 2000 to 2004. He was the Chair of Working Group 4 (New Radio Interfaces, Relay-Based Systems and Smart Antennas) of the Wireless World Research Forum (WWRF) in 2004 and 2005. He received the 2008 Canadian award for Telecommunications Research, a 2008 IEEE Technical Committee for Wireless Communications Recognition Award, and the IEEE Canada 2009 Fessenden Award (Telecommunications). He is an IEEE Life Fellow. 


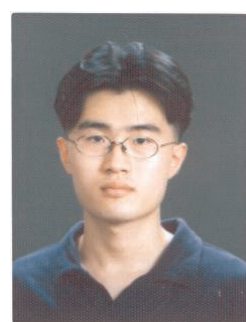

Young-Doo Kim (S'99-M'06) received the B.S., M.S., and Ph.D. degrees in electrical engineering from Korea Advanced Institute of Science and Technology (KAIST), Daejeon, Korea, in 1999, 2001, and 2006, respectively.

He has been with the Samsung Advanced Institute of Technology (SAIT), Gyeonggi-Do, Korea, since 2006. His primary research interests include synchronization, detection and estimation for communication systems, and predistortion linearization of nonlinear power amplifiers for wireless applications. His current research activities include radio resource management in multihop and relaying networks and interference management in cellular radio systems.

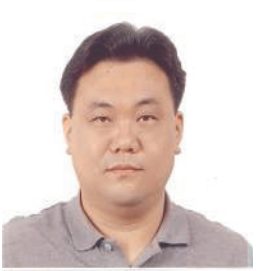

Eung Sun Kim (S'88-M'94) received the B.S., M.S. degrees in electronic communications engineering from Hanyang University, Seoul, Korea, in 1992, 1994 respectively. Since 2001 he is pursuing $\mathrm{Ph} . \mathrm{D}$. degree in Hanyang University.

$\mathrm{He}$ has been with the Samsung Advanced Institute of Technology (SAIT), Gyeonggi-Do, Korea, since 1994. His primary research interests include equalization and synchronization for communication systems. Currently, he is focusing on the radio resource management in multihop and relaying networks.
Yoon-Chae Cheong received the B.S. and M.S. degrees from Seoul National University, Korea, in 1981 and 1983, respectively, and the Ph.D. degree from Texas A\&M University, United States, in 1992.

He began his engineering career at the Electronics and Telecommunications Research Institute (ETRI) by working on the national project of developing the first digital exchange in Korea. From 1992 through 1997, he was with Hyundai Electronics Korea and Hyundai Electronics America, where he joined the team developing the CDMA cellular system. He was with Nortel Networks from 1998 to 2003 and participated in the development of the B3G air interface MAC/RRM algorithms. Since 2003, he has joined Samsung Advanced Institute of Technology (SAIT), where he contributed as team leads for modem platform, modem algorithm and systems engineering. Now, he is the vice president and team leader of advanced modem technology in Communications Lab of SAIT. 